\title{
Deep Chandra observations of diffuse hot plasma in M83
}

\author{
Q. Daniel Wang ${ }^{1 \star}$, Yuxuan Zeng ${ }^{1,2,3,4}$, Ákos Bogdán ${ }^{5}, \&$ Li Ji ${ }^{2,3}$ \\ ${ }^{1}$ Department of Astronomy, University of Massachusetts, Amherst, MA 01003, USA \\ ${ }^{2}$ Purple Mountain Observatory, Chinese Academy of Sciences, People's Republic of China \\ ${ }^{3}$ Key Laboratory of Dark Matter and Space Astronomy, CAS, People's Republic of China \\ ${ }^{4}$ Kapteyn Astronomical Institute, University of Groningen, PO Box 800, 9700 AV Groningen, The Netherlands \\ ${ }^{5}$ Harvard-Smithsonian Center for Astrophysics, 60 Garden Street, Cambridge, MA 02138, USA
}

Accepted 2021 October 12. Received 2021 September 30; in original form 2021 August 12

\begin{abstract}
It is widely believed that galaxy formation and evolution is regulated by stellar mechanical feedback in forms of fast stellar winds and supernova explosions. However, the coupling of this feedback with the interstellar medium remains poorly understood. We examine how the coupling may be traced by diffuse soft X-ray emission in M83 - a nearby face-on spiral galaxy undergoing active star formation, based chiefly on 729 ks Chandra observations. Our main findings are 1) the X-ray emission is enhanced not only along the galaxy's grand spiral arms, but also clearly in their downstreams; 2) the spectrum of the emission can be well characterized by a super-solar metallicity plasma with a lognormal temperature distribution, plus an X-ray absorption of a lognormal column density distribution; 3 ) the intensity of the emission is strongly anti-correlated with the dust obscuration seen in optical images of the galaxy. These findings suggest A) the morphology of the X-ray emission is likely due to the convolution of the feedback heating of the plasma with its thermal and dynamical evolution; B) the X-ray emission, accounting for $\sim 10 \%$ of the feedback energy input rate, probably traces only the high-energy tail of the radiation from the plasma; C) a good fraction of the recent star forming regions seems sufficiently energetic to produce multi-phased outflows, likely responsible for much of the dust obscuration and X-ray absorption. Direct confrontation of the findings with theories/simulations could help to understand the underlying astrophysics of the coupling and how the hot plasma shapes the interstellar medium.
\end{abstract}

Key words: galaxies: general, ISM, spiral, ISM: general, X-rays: general, ISM

\section{INTRODUCTION}

Stellar feedback is an important part of our present understanding of galaxy formation and evolution (e.g., Hopkins et al. 2014; Fujimoto et al. 2016; Kelly et al. 2020). However, much of this understanding is still not based on first principles, rather on recipes for subgrid astrophysics, which have been subject to few direct observational tests, leaving us with great uncertainties as to how the feedback operates and which prescription of physics might be closest to reality. (e.g., Somerville \& Davé 2015; Li et al. 2020; Hopkins et al. 2021; Quataert et al. 2021; Afruni et al. 2021; Jeffreson et al. 2021). We do have a solid framework for the feedback from high-mass stars in the form of fast stellar winds, core-collapsed supernovae (CC$\mathrm{SNe}$ ) and radiation, although its coupling with the surrounding interstellar medium (ISM) is poorly understood. The coupling cannot be well simulated, because of its multiphase nature and enormous scale range from individual stars to galaxies and beyond to the intergalactic medium. Therefore, the true impact of the stellar feedback on galaxy formation and evolution remains uncertain.

Diffuse X-ray emission has commonly been used to trace the mechanical feedback from stars. Much of the mechanical energy from fast stellar winds and $\mathrm{SNe}$ is expected to be thermalized, at least ini-

\footnotetext{
* Contact e-mail:wqd@umass.edu
}

tially, in diffuse hot plasma (e.g., Tang \& Wang 2005; Tang et al. 2009; van de Voort et al. 2016). Assuming that the emission arises from this plasma in an optically-thin collisional ionization equilibrium state, one may estimate its mass, energy, and metallicity (e.g., Wang et al. 2001; Tyler et al. 2004; Doane et al. 2004; Wang 2010; Li \& Wang 2013). However, such estimation sensitively depends on the assumed temperature distribution of the plasma, as well as its volume filling and foreground $\mathrm{X}$-ray absorption properties. This dependence has not been carefully addressed in existing studies. The spectral shape of the diffuse soft X-ray emission in individual galaxies is poorly characterized in general, typically with different combinations of discrete temperature components. Such combinations are highly degenerate and somewhat arbitrarily chosen. As a result, comparison of results from different analyses is often hardly practical or useful (e.g. Kuntz \& Snowden 2010).

Nearby face-on spiral galaxies provide us with the birds eye views of the diffuse X-ray emission and its relationship to stellar and ISM distributions in galactic disks. Early Chandra studies, typically with only moderate exposures $(<100 \mathrm{ks})$, have shown strong correlation between diffuse X-ray and $\mathrm{H} \alpha$ emissions (e.g., Tyler et al. 2004; Doane et al. 2004; Wang 2010). The tight association of the X-ray emission with spiral arms is somewhat unexpected, because the mechanical energy output rate from SNe should be nearly constant over a time duration $\left(\sim 3.2 \times 10^{7}\right.$ yrs or the lifetime of $\sim 8 \mathrm{M}_{\odot}$ stars that 
will eventually end in core-collapse SNe; McCray \& Kafatos 1987) that is much longer than the lifetime of the stars responsible for $\mathrm{H} \alpha$ emission $\left(\sim 5 \times 10^{6} \mathrm{yrs}\right)$. This time duration is a considerable fraction of the orbit period of stars around galactic centers. Furthermore, the bulk of the mechanical feedback energy is missing from the Xray detection (e.g., Tyler et al. 2004; Wang 2010; Wang et al. 2016).

In recent years, deep Chandra observations have been obtained for a few nearly face-on galaxies. However, studies based on these data mostly focus on individual discrete sources or their populations. One clear exception is the study of the diffuse X-ray emission in M101 (Kuntz \& Snowden 2010, KS10 hereafter). This comprehensive work shows that the bulk ( $\gtrsim 80 \%$ ) of the emission is associated with star forming regions with ages $\lesssim 20 \mathrm{Myr}$, as traced by FUV emission, although the one-to-one correlation between X-ray and FUV "knots" is not strong. Furthermore, the X-ray spectrum in the diffuse emission dominant band $(\lesssim 1 \mathrm{keV})$ appears to depend on local environment; statistically, regions with higher $\mathrm{X}$-ray intensities tend to have harder spectra. One would wonder whether or not diffuse X-ray emission in other galaxies behaves similarly and if its origin and evolution could be better understood.

Similar studies on diffuse X-ray emission from face-on spiral galaxies have been carried out using XMM-Newton observations (e.g., Owen \& Warwick 2009; Weżgowiec et al. 2020). Their relatively large field coverage and sensitivity allow to map out the global distribution and to conduct spectral analysis of this emission. Indeed, the studies show $0.2-2 \mathrm{keV}$ emission permeating the galactic disks. With the limited spatial resolution of the XMM-Newton observations, however, confusion with discrete sources, including background AGNs and clusters of galaxies, is considerable and the separation of spiral arms and inter-arm regions is difficult. A complementary high spatial resolution examination of the X-ray emission remains essential to determine the intrinsic spatial, thermal and chemical properties of the diffuse hot plasma.

Here we present a detailed analysis of M83 [NGC 5236; centered at R.A. $=13^{h} 37^{m} 00^{\mathrm{s}} 95$; Decl. $=-29^{\circ} 51^{\prime} 55^{\prime \prime} 5$ (J2000.0), NED], based on archival Chandra observations (Fig. 1). Classified as SBc(s) II, at the distance $D=4.5 \mathrm{Mpc}\left(1^{\prime} \equiv 1.3 \mathrm{kpc}\right.$, Thim et al. 2003) and having a low foreground $\mathrm{HI}$ column density $\left(\mathrm{N}_{\mathrm{H}}=4.1 \times 10^{20} \mathrm{~cm}^{-2}\right.$ (HI4PI Collaboration et al. 2016), this face-on grand-spiral galaxy (disk inclination $\sim 15^{\circ}$ ) is well-suited for the study of diffuse $\mathrm{X}$-ray emission and its relationship to the stellar feedback. Fig. 1A shows the coverage of the Chandra observations, focusing on the on-axis back-illuminated S3 chip. A total of 729 ks exposure is used in the study of discrete X-ray sources of the galaxy, as well as its bright starburst nucleus (Long et al. 2014), which is not included in the present study. Following Foyle et al. (2012), we assume a trailing spiral structure of the galaxy, rotating in the clockwise direction and having the co-rotation radius of $r_{c}=3.7 \mathrm{kpc}$ (or $2 ! 8$ ). For ease of reference, we trace the leading edges of the two major spiral arms in Fig. 1B. The edges, apparent in high-resolution CO maps, represent the locations of coherent dust lanes seen in optical images.

The rest of this paper is organized as follows. We describe the Chandra observations and data reduction/analysis procedures in $\S 2$. After presenting results of the analysis in $\S 3$, we discuss their implications in $\S 4$. Finally, in $\S 5$, we summarize the major results and conclusions of the present study. Following KS10, we express the intensity of the diffuse X-ray emission as photons $\mathrm{cm}^{-2} \mathrm{~s}^{-1} \operatorname{arcsec}^{-2}$, which will be referred to as surface brightness units (abbreviated as sbu). We present measurement errors (or uncertain intervals) of model parameters at the $90 \%$ confidence.

\section{OBSERVATIONS AND DATA REDUCTION/ANALYSIS}

\subsection{Chandra data}

\subsubsection{X-ray data selection and calibration}

We use the same 10 Chandra observations as those used in Long et al. (2014). They were all taken with the Advanced CCD Imaging Spectrometer (ACIS). M83 was mainly covered by the backilluminated S3 chip (Fig. 2). The exposure map shown in Fig. 1A includes the effective area of the telescope plus the instrument. For simplicity and uniformity, we chiefly use the data collected by this chip for our on-galaxy data analysis, although the "off-galaxy" Xray background is estimated with the data from the adjacent frontilluminated S2 chip of the observation \# 12994, which had the longest exposure $(150 \mathrm{ks})$ among the observations.

We reprocess the data with the standard pipeline of the Chandra Interactive Analysis of Observations (CIAO; version 4.12 CALDB 4.9.2.1), including the light curve cleaning with the routine LC_CLEAN. We construct the mosaic count and exposure maps in individual bands.

\subsubsection{Source detection and exclusion}

Using the merged maps and their position-dependent and weighted point spread function (PSF), we detect discrete sources, using WAVEDETECT on the scales of 1.0, 1.4, 2.0, 2.8, 4.0, 5.7, and 8.0 pixels $(0$." $492 \times 0$." 492 each) and in the soft $(0.3-1.5 \mathrm{keV})$, hard $(1.5-7 \mathrm{keV})$ and broad $(0.5-7 \mathrm{keV})$ bands. The detected sources are merged and are visually examined for their integrity (Figs. 2 and $3 \mathrm{~A}$ ). The source detection limit is $\lesssim 10^{36} \mathrm{erg} \mathrm{s}^{-1}$ in the $0.35-8 \mathrm{keV}$ band (assuming the distance to M83), depending on the X-ray absorption, local X-ray background and effective exposure, which are all position-dependent (see also Long et al. 2014).

To examine the diffuse X-ray emission, we exclude regions significantly contaminated by the detected sources. For relatively faint sources ( $\gtrsim 0.01$ counts s ${ }^{-1}$ ), excluding the $90 \%$ energy-encircled ellipses is sufficient. For brighter source, we manually increase the region sizes to remove visible ring-like features due to the PSF wing. In the present study, we also remove both the central region $\left(24^{\prime \prime}\right.$ radius) of M83 and the region (18" radius) around a background cluster of galaxies (Figs. 2 and 3A; Long et al. 2014, see also Appendix A). We estimate the contamination from the PSF spill-out of the discrete sources to be $\lesssim 5 \%$ of the net diffuse flux in the $\lesssim 1 \mathrm{keV}$ band, which is mostly considered here; the contamination can be significant, however, in higher energy bands and especially in regions close to relatively bright sources. Another contribution that is not related to diffuse hot plasma is faint X-ray binaries below our detection limit. Their luminosity function is known to be rather flat, although its exact shape at the lower luminosity end remains uncertain, because of the statistical (Eddington) bias in the source detection (Wang 2004), as well as such contamination as supernova remnants and background AGN, which are subject to X-ray absorption in M83. But according to Long et al. (2014), the index of the cumulative luminosity function of discrete sources in M83 appears to be $\lesssim 0.5$, as expected for low-mass X-ray binaries, which tend to dominate at low luminosities $\left(\lesssim 10^{37} \mathrm{erg} \mathrm{s}^{-1}\right)$ in the Chandra energy range. We estimate that the X-ray binary contribution below the detection limit should be smaller than the PSF spill-out contamination of the detected discrete sources.

For the visualization of the diffuse (source-removed) emission only, we fill the holes left from the source exclusion. The filling uses counts randomly selected from events in surrounding regions 

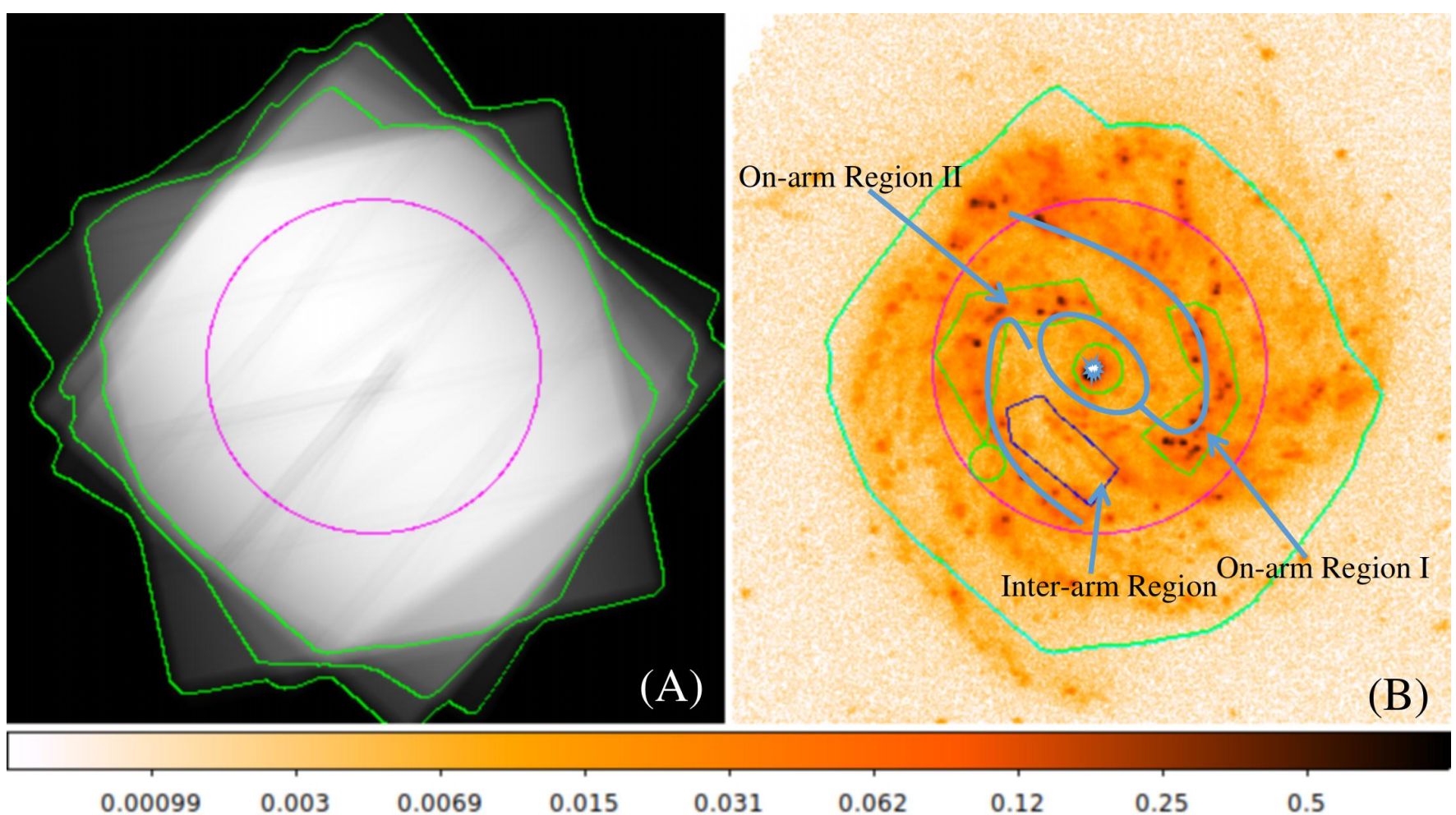

0.003

0.0069

0.015

0.031

0.062

0.12

0.25

0.5

Figure 1. Overview of the Chandra overage of M83: (A) the effective exposure map of the on-axis $\mathrm{S} 3$ chip in the $0.45-1 \mathrm{keV}$ band, together with the exposure contours at $(0.1,0.5$ and 1$) \times 10^{8} \mathrm{~s} \mathrm{~cm}^{2}$; (B) the $10^{8} \mathrm{~s} \mathrm{~cm}^{2}$ contour (colored in agua), $\sim 50 \%$ of the maximum exposure of the map, overlaid on GALEX FUV image of M83, together with an ellipse and two curves (all in blue gray) illustrating the central bar and leading edges of the grand-spiral arms of the galaxy. The two green circles outline the fields where the very central region of the galaxy and the presence of a background galaxy cluster (see Appendix A) dominate the observed X-ray emission; these fields are excluded from our quantitative analysis of the source-removed "diffuse" X-ray emission. Also marked in both panels is the galaxy's co-rotation radius of $r_{c}=3.7 \mathrm{kpc}$ (or 2! 8 ; Foyle et al. 2012; the magenta-colored circle). The outer disk spectrum of the emission is extracted from the field outside $r_{c}$, but within the exposure contour, whereas the inner disk spectrum is from within $r_{c}$; in addition, sample on-arm and inter-arm spectra are extracted from regions outlined by the green and deep blue polygons (see Table 1).

of the individual sources, using the ciao routine ROI and DMFILTH (Fig. 3B). The source-removed regions are not used in the quantitative analysis of the emission (e.g., the construction of the radial intensity profile or spectra).

\subsubsection{Background Estimation}

The background consists of two components: non-X-ray (cosmicray-induced) events and cosmic X-rays. We estimate the non-X-ray background, using data taken while the instrument was in the stowed position, out of the focal plane and under a shield ${ }^{1}$. For each observation, we use stowed events of the same chips to estimate the non$\mathrm{X}$-ray background, the level of which is adjusted so that it matches the count rate actually detected in the $10-12 \mathrm{keV}$ band, where Chandra has virtually no effective area for X-rays. This non-X-ray background contribution is subtracted from both imaging and spectral analyses.

To study the net X-ray emission from M83, we also need to estimate the local X-ray background, which is assumed to be uniform across the field. This estimation is based on modeling the spectrum constructed from counts detected in the "off-galaxy" S2 chip, after excluding detected sources and accounting the stowed background. We characterize the X-ray background spectrum with

\footnotetext{
1 http://cxc.harvard.edu/contrib/maxim/stowed/
}

the spectral model $m_{x b}=A P E C+T B A B S(A P E C+P O W E R L A W)$, where the names of the spectral model components are in the form used in the spectral analysis software XSPEC (which is part of HEASOFT v6.28). The first component, APEC, represents the contribution from optically-thin thermal plasma (Smith et al. 2001) of $k T=0.1 \mathrm{keV}$ (fixed) in the Local Bubble, while the second additive term, APEC + POWERLAW, is for the background emission from more distant diffuse hot gas (e.g., in the Galactic gaseous thick disk and halo) and unresolved point-like sources (primarily AGNs; assumed to have a power law spectrum) and are subject to an $\mathrm{X}$ ray absorption $(T B A B S)$ due to the Galactic foreground $\mathrm{HI}$ column density of $N_{H}=4.21 \times 10^{20} \mathrm{~cm}^{-2}$. The solar elemental abundances (aspl in XSPEC) are assumed. These assumptions and physical representations about the individual components are not important, as long as the best-fit model as a whole gives a sufficiently accurate characterization of the overall spectral shape of the sky background. With the model, we estimate the X-ray background contribution in any on-galaxy region, spectrally or in a broad band, accounting for the area difference in the spectral extraction, as well as the instrument response difference between the two chips (back-illuminated vs. front-illuminated). Specifically, the X-ray background contribution is $8.65 \times 10^{-10}$ sbu in the $0.45-1 \mathrm{keV}$ band $-\mathrm{a}$ band that is optimal for imaging the emission from diffuse hot plasma, accounting for the uncertainties in the instrument sensitivity and background and the point source contamination (KS10). This contribution is subtracted from our on-galaxy $0.45-1 \mathrm{keV}$ image of M83. 


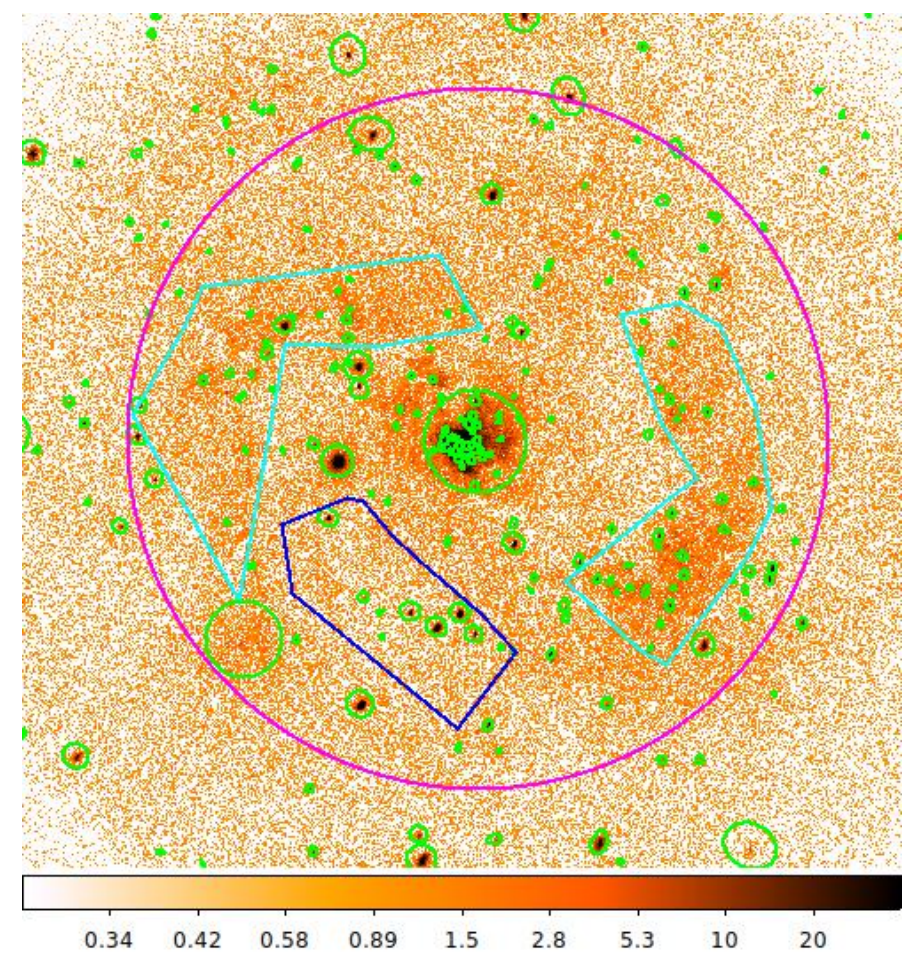

Figure 2. ACIS-S3 $0.45-7 \mathrm{keV}$ count mosaic of M83 constructed from the Chandra observations. Small green ellipses outline the averaged $90 \%$ energyencircled PSF regions of individual detected sources. The rest of the overlaid regions are the same as those in Fig. 1.

\subsubsection{Spectral Analysis}

With the limited spectral resolution of the ACIS data, our focus in the present work is to provide a relatively simple and uniform spectral characterization of the diffuse X-ray emission observed in various representative regions in M83 (e.g., Fig. 1B). We adaptively group each on-galaxy spectrum to achieve a $S / N>3$ per bin, where the specific spectral flux $S$ excludes the non-X-ray background contribution. With the local X-ray background contribution modeled in the off-galaxy field (see $\$ 2.1 .3$ ), the remaining emission likely represents a combination of diffuse hot plasma and an accumulated contribution from discrete sources below our detection limit, plus a small residual left from the removal of the detected ones. This latter source contribution, usually insignificant in the soft range $(\lesssim 1.5 \mathrm{keV})$, can be important at higher energies and can be adequately modeled with a power law with a fixed photon index $\Gamma=1.7$ (e.g., Lehmer et al. 2017). We assume that the diffuse soft X-ray emission is dominated by optically-thin thermal plasma in collisional ionization equilibrium and test various models, including single and multiple temperature ones. We find that a (variable abundance) lognormal temperature distribution model of the plasma emission measure (VLNTD or lognormal plasma hereafter) gives a simple and apparently more physical characterization of the diffuse hot plasma (see more discussion on this in $\S 4.3$ and Cheng et al. 2021). The temperature distribution of the emission measure in this lognormal plasma is determined by the two key parameters, the EMweighted mean temperature $\bar{x}=\ln (\overline{\mathrm{T}})$ and the temperature dispersion $\sigma_{x}$ in logarithm form. However, the fits of the lognormal plasma with a uniform foreground X-ray absorption to the inter-arm and inner disk spectra are not acceptable at $\gtrsim 3 \sigma$ confidences. Therefore, we further adopt a multiplicative model LNABS, which is simply a sum of the foreground X-ray absorption weighted by a lognormal distribution of the absorbing gas column density [see Appendix C of Cheng et al. (2021)], which will be referred as the lognormal absorption model hereafter. This model accounts for the intrinsic absorption in M83, which is in addition to a fixed uniform Galactic foreground absorption. The inclusion of the model significantly improves the fits to all the spectra (Table 1); each of them cannot be rejected statistically at a confidence $\gtrsim 2 \sigma$.

\subsubsection{Imaging analysis}

We produce the background-subtracted and exposure-corrected intensity images of the diffuse soft X-ray emission from M83 in various bands. Consistent with the approach used in KS10, our imaging analysis of the diffuse X-ray emission is largely restricted to the ACIS-S3 $0.45-1.0 \mathrm{keV}$ range. For presentation only, we smooth the intensity mosaic with either CIAO CSMOOTH, which adaptively adjusts the smoothing scale to achieve a signal-to-noise ratio $>3$ (Fig. 3), or a Gaussian (e.g., Fig. 4).

With the local X-ray background subtracted, we further construct hardness ratio (HR) maps of the on-galaxy emission, as detailed in Appendix B. The HR is defined as the ratio of the emission intensities in the two bands: $\mathrm{I}(0.7-1 \mathrm{keV}) / \mathrm{I}(0.45-0.7 \mathrm{keV})$, same as that used by KS10. The $0.45-0.7 \mathrm{keV}$ and $0.7-1.0 \mathrm{keV}$ bands are dominated by the OVII triplet plus the OVIII line and the Fe-L complex, respectively. The two images are smoothed with a Gaussian of $\sigma=7$ "' 87 before the division to form the HR map. However, all our quantitative calculations, including all 1-D presentations (radial and other 1-D intensity profiles), are based on data without any such smoothing.

\subsection{Auxiliary data}

We employ multi-wavelength data to trace various stellar and interstellar components of M83. Some of such data are adopted only for image overlay comparison, while others are included in more quantitative comparisons. Downloaded from the Multi-Mission archive at the Space Telescope Science Institute (MAST) ${ }^{2}$, the GALEX FUV image (Figs. 1B and 4B) is mostly sensitive to the emission from young massive stars. The image intensity is in units of counts $\mathrm{s}^{-1}$ pixel $^{-1}$, where the pixel has a size of $1 .{ }^{\prime \prime} 5^{3}$. Similarly obtained are also various HST WFC3/UVIS mosaick images ${ }^{4}$, which provide us with high-resolution optical views of M83. Specifically, we use the narrow-band $\mathrm{F} 657 \mathrm{~N}(\mathrm{H} \alpha)$ image to trace recent massive star forming regions (Fig. 5) and the broad band F438W (B-band), F555W (V-band) and F814W (I-band) images to mainly probe dust attenuation (Fig. 6). For example, we construct a color index (CI) map with the F438W and F814W images. To do so, we first estimate the local background (including both sky and instrument contributions) in an "off-galaxy" region (Fig. 6) and then convert the background-subtracted images to the magnitude ones $\left(m_{F 814 W}\right.$ or $\left.m_{F 438 W}\right)$. The CI in the resultant map $\left(m_{F 438 W}-m_{F 814 W}\right)$ provides a proxy of the dust attenuation and hence the foreground X-ray absorption in the galaxy.

The ALMA ${ }^{12} \mathrm{CO} \mathrm{J}=2-1$ line emission data on M83 are obtained as part of the PHANGS-ALMA survey ${ }^{5}$ (Leroy et al. 2021). We here use only the moment0 mosaic map (which combines ALMA's main

\footnotetext{
2 https://archive.stsci.edu/missions-and-data/galex

3 http://www.galex.caltech.edu/researcher/data.html

4 https://archive.stsci.edu/hst/

5 https://www.canfar.net/storage/list/phangs/RELEASES
} 

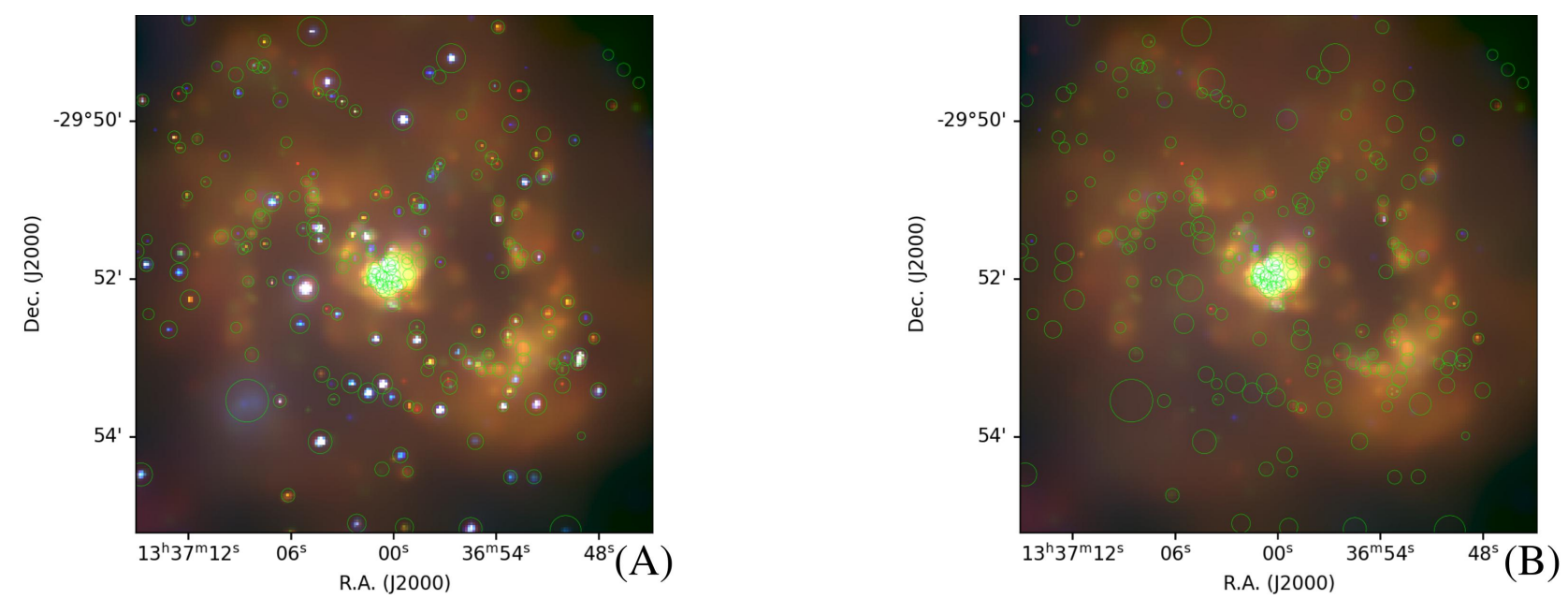

Figure 3. (A) 3-color Chandra intensity montage of M83 in the ACIS-S3 bands: $0.3-0.7 \mathrm{keV}$ (red), 0.7-1.5 keV (green) and $1.5-7 \mathrm{keV}$ (blue). The marked discrete sources are the same as those in Fig. 2. (B) Same as in (A), but with the sources excised. Images in individual bands are adaptively smoothed.
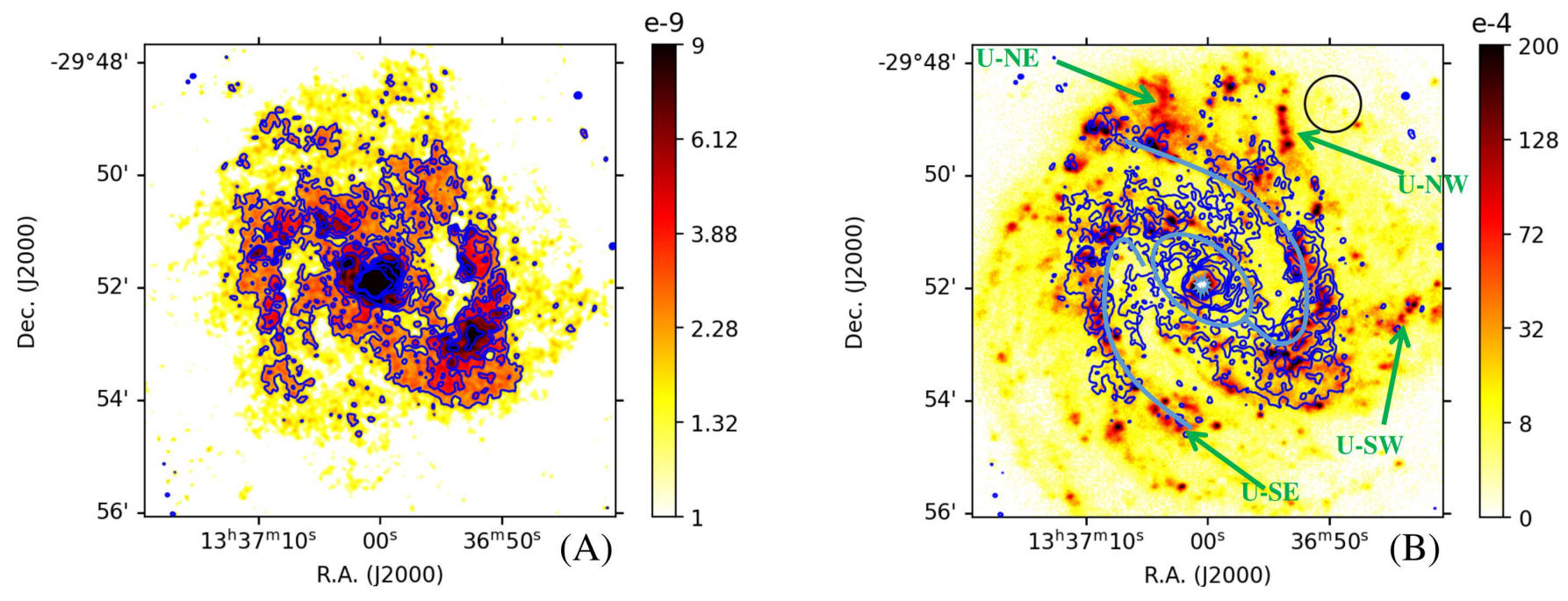

Figure 4. (A) Intensity image of the diffuse X-ray emission in the $0.45-1 \mathrm{keV}$ band, which is smoothed with a Gaussian of $\sigma=3$ !" 9 . The contour levels are at $\left(1.3,2.6,4.2,8.4,17\right.$, and 34) $\times 10^{-9}$ sbu. (B) The same contours overlaid on the GALEX FUV image of M83. From the image, a local background has been subtracted, which is estimated in the field outlined by the upper right circle. Also marked are a few prominent FUV peaks that do not show corresponding X-ray enhancements in this contour plot. The illustration lines are the same as those in Fig. 1B.

12-m array, the 7-m array, and total power observations) to trace dense molecular gas in the galaxy at a resolution of $\sim 1^{\prime \prime}$ (Fig. 5).

We further use 2MASS K-band and WISE $22 \mu \mathrm{m}$ band images to model the distributions of accumulated stellar mass $\left(M_{*}\right)$ and recent star formation rate (SFR) in M83. Our interest here is primarily in the relationship of the diffuse soft X-ray intensity to the feedback from massive stars, which is proportional to the surface (area specific) SFR, while accounting for the contribution from faint sources (mostly cataclysmic variables and other active binaries), which is proportional to the stellar mass.

\subsubsection{Estimation of the stellar mass $X$-ray contribution}

To estimate $M_{*}$ from the $2 M A S S$ K-band luminosity $L_{K}$ (both in solar units), we use the relation (Bell \& de Jong 2001)

$$
\log \left(M_{*} / L_{K}\right)=-0.692+0.652(B-V),
$$

which, together with the optical color $B-V=0.59$ (NED) for M83, gives the mass-to-light ratio as $0.493 M_{\odot} / L_{K, \odot}$. We further use the relation (Ge et al. 2015)

$$
L_{0.45-1 \mathrm{keV}} / M_{*}=5.3 \times 10^{27} \mathrm{ergs}^{-1} \mathrm{M}_{\odot}^{-1}
$$

to obtain the conversion to the $0.45-1 \mathrm{keV}$ X-ray luminosity

$$
L_{0.45-1 \mathrm{keV}}=2.6 \times 10^{27}\left(L_{K} / L_{K, \odot}\right) \mathrm{erg} \mathrm{s}^{-1} .
$$

To apply the above conversion to the 2MASS intensity image ( $I_{K}$ in units of $\mathrm{DN} \mathrm{s}^{-1} \operatorname{arcsec}^{-2}$ ), we first calculate the surface magnitude as

$$
m_{K}=19.9-2.5 \log \left(I_{K}\right),
$$

where 19.9 is the magnitude zero point (or the KMAGZP parameter value in the fits header of the image), and then adopt the absolution K-band magnitude of the Sun as 3.28 to obtain the surface luminosity of the image as

$$
L_{K} / L_{K, \odot}=10^{-0.4\left[m_{k}+5-3.28-5 \log \left(D_{p c}\right)\right]} .
$$



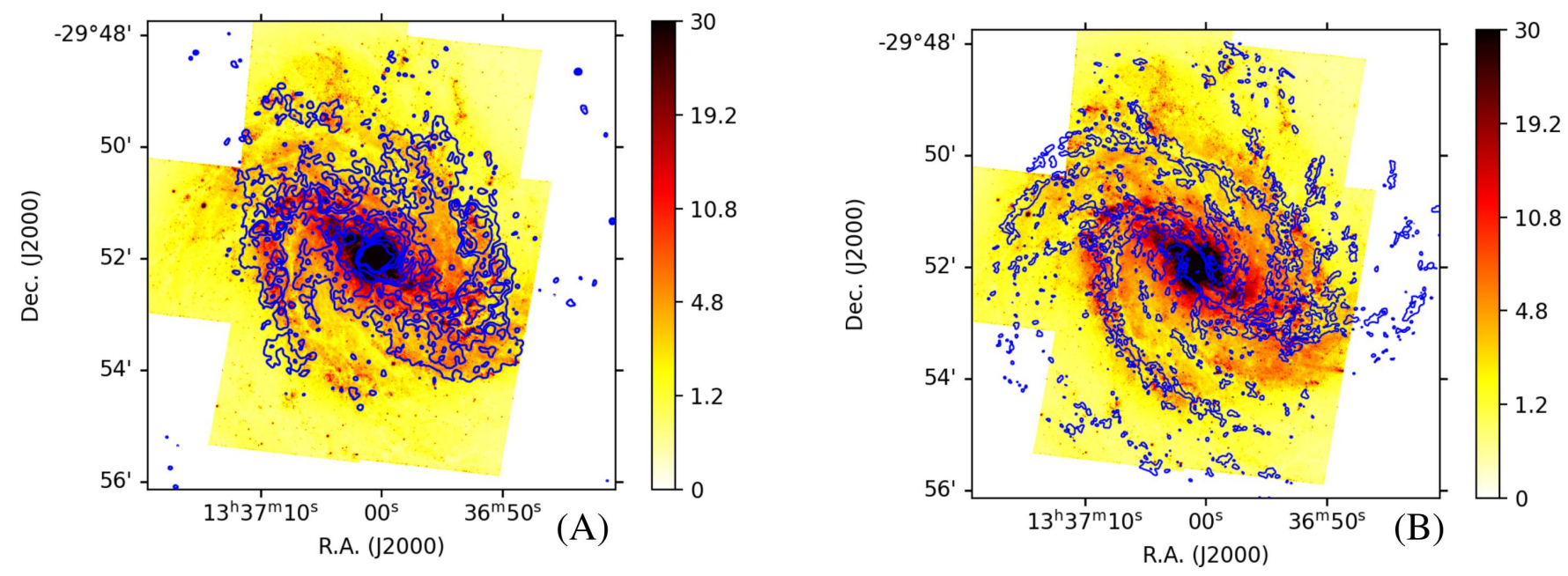

Figure 5. HST $\mathrm{H} \alpha$ image overlaid with the X-ray intensity contours same as in Fig. 4 (A) or with the ALMA ${ }^{12} \mathrm{CO} \mathrm{J}=2-1$ intensity contours at 10 and 50 $\mathrm{K} \mathrm{km} \mathrm{s}^{-1}$ (B). These plots demonstrate that the molecular line emission is strongly concentrated along prominent dust lanes or on their slightly downstream sides of the spiral arms. Both the $\mathrm{H} \alpha$ and $\mathrm{X}$-ray emissions extend further toward the downstream sides.
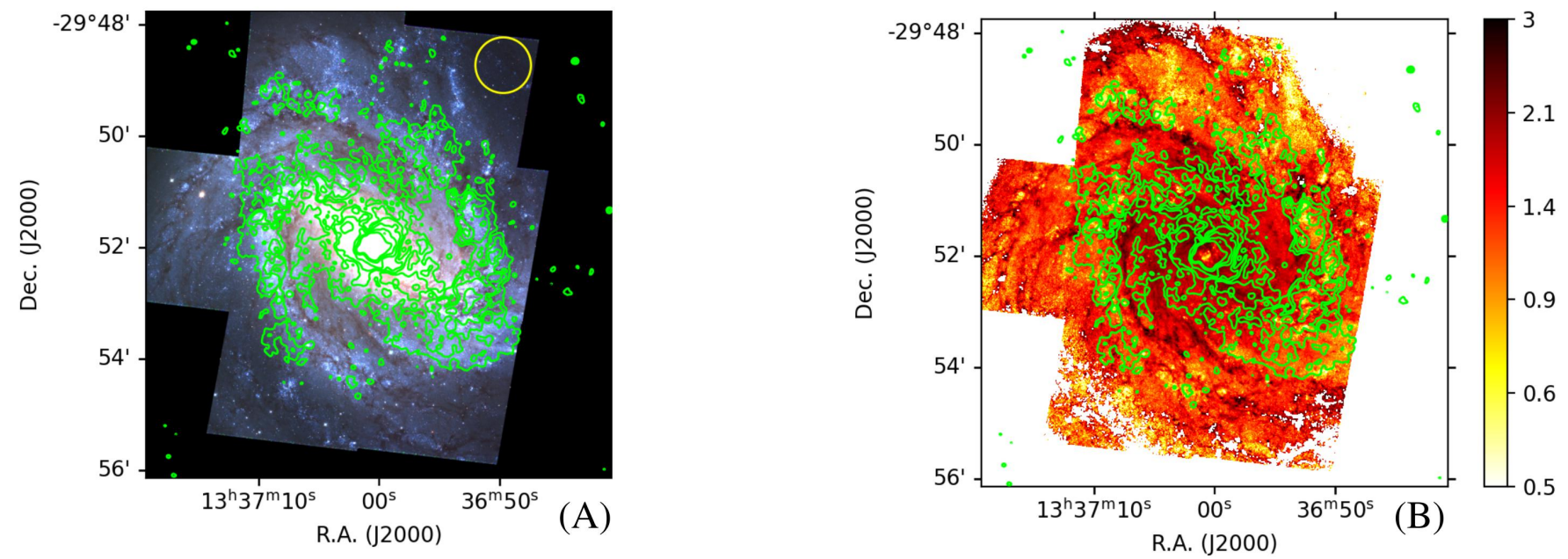

Figure 6. X-ray intensity contours same as in Fig. 4 are overlaid on the HST 3-color composite of B-band (blue), V-band (green) and I-band (red) images (A) and the HST B-I color-index image (B). Anti-correlation of the X-ray intensity with the dust extinction is apparent. The yellow circle in (A) marks the region used to calculate the local background of the HST images.

With $L_{0.45-1 \mathrm{keV}}$ from Equation 3, we can express the diffuse X-ray intensity as $S_{x, *}=a L_{0.45-1 \mathrm{keV}} /\left(4 \pi D^{2}\right)$, where $a=1.1 \times$ $10^{9} \mathrm{sbu} /\left(\mathrm{erg} \mathrm{s}^{-1} \mathrm{~cm}^{-2} \operatorname{arcsec}^{-2}\right)$ is the conversion from the energy intensity to the on-axis sbu in the $0.45-1 \mathrm{keV}$ band, assuming the best-fit spectral model for the inner disk in Table 1. Since we are applying the above derivation to the stellar K-band intensity distribution (i.e., using $I_{K}$ ), the units of $L_{K}, M_{*}$, and $L_{0.45-1 \mathrm{keV}}$ should all be considered to be per $\operatorname{arcsec}^{2}$. Putting all these together, we get

$$
S_{x, *}=\left(5.3 \times 10^{-11} \mathrm{sbu}\right) I_{K}
$$

\subsubsection{Surface star formation rate as the stellar feedback tracer}

There are various methods to estimate the surface SFR $\left(\Sigma_{S F R}\right)$ in a nearby star-forming galaxy (e.g., using the FUV+IR emission; Leroy et al. 2008; Liu et al. 2013). We here adopt the simply relation be- tween the $22 \mu \mathrm{m}$ spectral luminosity $\left(L_{22}\right)$ and SFR (Jarrett et al. 2013)

$$
\operatorname{SFR}\left(\mathrm{M}_{\odot} \mathrm{yr}^{-1}\right)=7.50 \times 10^{-10} L_{22}\left(L_{\odot}\right)
$$

where the total solar luminosity $L_{\odot}=3.839 \times 10^{33} \mathrm{erg} \mathrm{s}^{-1}$. Jarrett et al. (2013) show that the total SFR of M83 estimated this way is $2.8 \mathrm{M}_{\odot} \mathrm{yr}^{-1}$, consistent with those from the $24 \mu \mathrm{m}\left(2.8 \mathrm{M}_{\odot} \mathrm{yr}^{-1}\right)$ and the combined FUV+IR $\left(3.2 \mathrm{M}_{\odot} \mathrm{yr}^{-1}\right)$ methods. Following Vargas et al. (2018), we here use the above relation to estimate $\Sigma_{S F R}$ (in units of $\mathrm{M}_{\odot} \mathrm{yr}^{-1} \operatorname{arcsec}^{-2}$ ) of M83 from now the $22 \mu \mathrm{m}$ surface spectral luminosity $L_{22}$. This luminosity distribution can be related to a WISE $22 \mu \mathrm{m}$-band intensity image ( $I_{22}$ in units of $\mathrm{DN} \mathrm{s}^{-1} \operatorname{arcsec}^{-2}$ ), in which, the unresolved stellar contribution in M83, as well as foreground stars, have been excised (Jarrett et al. 2019). Although the effective angular resolution of this image is quite limited (11."9 FWHM), the availability of the WISE all-sky survey makes it attractive to use and easy for comparison with other 


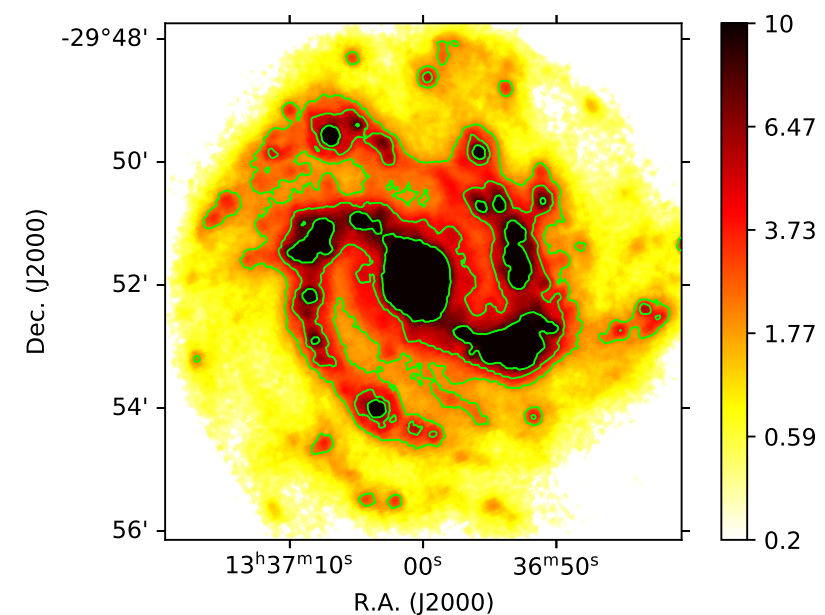

Figure 7. Processed WISE $22 \mu \mathrm{m}$ image after the subtraction of a local background (25 DN s${ }^{-1} \operatorname{arcsec}^{-2}$ ), as well as foreground stars (Jarrett et al. 2019). The contours are at $\left(0.5,1\right.$ and 2) $\times 4.3 \mathrm{DN} \mathrm{s}^{-1} \operatorname{arcsec}^{-2}-$ the level tracing the surface SFR threshold for outflows (§ 4.2).

studies. The resolution is sufficient, considering that the SFR estimation methods are all calibrated on scales $\gtrsim 1 \mathrm{kpc}$.

$L_{22}\left(\mathrm{~L}_{\odot} \operatorname{arcsec}^{-2}\right)$ is related to $v I_{22}\left(\right.$ where $v=1.3 \times 10^{13} \mathrm{~Hz}$ is adopted, corresponding to the central wavelength of $22.8 \mu \mathrm{m}$ of the WISE band; Brown et al. 2014) via

$$
\begin{aligned}
L_{22} & =1.3 \times 10^{13} \times 5.2 \times 10^{-5} I_{22}\left(4 \pi D^{2}\right) /\left(10^{23} \times 3.8 \times 10^{33}\right) \\
& =4.4 \times 10^{3} I_{22},
\end{aligned}
$$

where the term $5.2 \times 10^{-5}$ converts $\mathrm{DN} \mathrm{s}^{-1}$ to Jy (see Explanatory Supplement to the WISE All-Sky Data Release Products ${ }^{6}$ ) and the distance to M83 is adopted. Combining the above two equations, we obtain

$$
\Sigma_{S F R}\left(\mathrm{M}_{\odot} \mathrm{yr}^{-1} \operatorname{arcsec}^{-2}\right)=3.3 \times 10^{-6} I_{22}\left(\mathrm{DN} \mathrm{s}^{-1} \operatorname{arcsec}^{-2}\right) .
$$

We do not attempt here to match the resolution of the surface stellar mass or SFR model image to that of a particular diffuse soft X-ray intensity image. This latter image is very much limited by counting statistics, especially at a low surface brightness level, and is typically presented at a spatial resolution lower than those of these relevant multi-wavelength images. In M83, the stellar mass is not particularly structured and contributes weakly to the diffuse soft X-ray intensity. So the resolution should not be a significant issue. Some caution is needed in the comparison of the X-ray data with the surface SFR, which is certainly more structured (Fig. 7). So when desirable, we also invoke an Spitzer $24 \mu \mathrm{m}$ image with a resolution of $\sim 6^{\prime \prime}$ (FWHM; Dale et al. 2009), downloaded from NED.

\subsection{1-D Decomposition of the diffuse soft X-ray intensity}

Using the stellar mass and SFR distributions estimated above, we may approximately decompose the observed diffuse soft X-ray intensity of M83 into two components: 1) the integrated unresolved stellar contribution $\left.\left(S_{x, *}\right) ; 2\right)$ emission traced by $b \Sigma_{S F R}$, where the

\footnotetext{
${ }^{6}$ https://wise2.ipac.caltech.edu/docs/release/allsky/ expsup/
}

coefficient $b$ [in units of $\mathrm{sbu} /\left(\mathrm{M}_{\odot} \mathrm{yr}^{-1} \operatorname{arcsec}^{-2}\right)$ ] is to be determined. This decomposition is conducted with the model radial intensity profile (in sbu):

$$
S_{x, m}(r)=S_{x, *}(r)+b \Sigma_{S F R}(r),
$$

where $S_{x, *}(r)$ and $\Sigma_{S F R}(r)$ are estimated with the corresponding imaging data (Equations 6 and 7). We fit $S_{x, m}(r)$ to the observed radial surface intensity profile of the diffuse soft X-ray emission $S_{x}(r)$ to obtain $b$. Again, our focus here is not on an accurate modeling of the diffuse soft X-ray emission, but is instead on its systematic positional deviation from the model to provide insights into the heating/cooling and dynamics of the underlying hot plasma in the disk of M83.

\section{RESULTS}

Fig. 2 presents the merged count image of M83 in the Chandra/ACIS-S3 $0.45-7 \mathrm{keV}$ band and with the detected sources marked. Fig. 3 shows an adaptively smoothed multi-band composite of the (stowed background-subtracted and exposure-corrected) $\mathrm{X}$-ray intensity images with or without detected sources excised. A Gaussian smoothed version of the intensity image of the diffuse Xray emission in the $0.45-1 \mathrm{keV}$ band is given in Fig. 4 . We compare the X-ray intensity contours with those observed in other wavelength bands, including the GALEX FUV image (Fig. 4B), mostly sensitive to massive stars; the HST $\mathrm{H} \alpha$ image (Fig. 5A), tracing recent starforming regions; and the HST broad band images (Fig. 6), revealing dust lanes. Fig. 5B further compares the $\mathrm{H} \alpha$ image with ${ }^{12} \mathrm{CO} \mathrm{J}=2-1$ intensity contours, depicting a positional relationship of recent massive star-forming regions with dense molecular gas, especially along the spiral arms (see further discussion in $\S 4$ ).

In Appendix A, we describe the properties of two extended background X-ray sources and their potential utilities for studying M83. One of these two sources is a distinct background cluster of galaxies, which is apparent in Fig. 3A as an extended hard (bluish) feature projected in the southeastern (SE) part of the galaxy. This cluster is removed in the construction of the diffuse X-ray emission images. The other source is represented by a FR II radio galaxy (e.g., Figs. A1 and C1; Cowan et al. 1994; Maddox et al. 2006). The $\mathrm{X}$-ray emission from the galaxy forms a linear feature, although its exact extent remains a bit uncertain. In the following, we focus on the diffuse X-ray emission from M83 itself.

\subsection{Spatial properties of the diffuse $X$-ray emission}

The grand-design spiral plus bar structure of M83 (e.g. Jarrett et al. 2013 ) is apparent in the diffuse X-ray emission image (e.g., Fig. 4A). Fig. 4B shows a general correlation between the X-ray and FUV intensities along the spiral arms. This correlation is more quantitatively demonstrated in Fig. 8, where the data are binned according to the ranked values of the $\mathrm{x}$-axis parameter: i.e., in the present case, the diffuse X-ray intensity is calculated with the pixels in each of the GALEX FUV intensity intervals evenly divided over the presented ranges, beyond which too few pixels exist to conduct a meaningful averaging. The correlation is calculated separately for the two regions: inside or outside $r_{c}$. Both regions show a consistent squareroot dependence of the X-ray intensity on the FUV, as seen in M101 (KS10).

This non-linear dependence also manifests in the spatial correspondence between the diffuse X-ray and FUV intensity enhancements. While they trace each other well at the sharp leading edges of 
the spiral arms, the X-ray enhancement appears substantially more extended on their downstream sides than the FUV. Furthermore, the $\mathrm{X}$-ray enhancement tends to fill those so-called inter-arm fork/void regions, showing little dusty dense gas (Figs. 5-6), and also permeates in the bar (Fig. 4), where the FUV is not enhanced due to strong extinction (Jarrett et al. 2013). In the outer arm regions (near or outside $r_{c}$ ), multiple FUV peaks do not show much diffuse soft X-ray enhancements even at the $1.3 \times 10^{-9}$ sbu level; the most notable peaks (U-NE, U-NW, U-SW and U-SE) are marked in Fig. 4B. Although the background level of the diffuse X-ray emission, which generally decreases with the off-center radius, is a significant factor in determining the weak X-ray appearance of the FUV peaks at the outer radii (see also KS10), other ISM effects also likely play a contributing role (see further discussion in $\S 4.1$ ).

Figs. 4-5 further demonstrate the relationship among multiwavelength components in and around the spiral arms. At the level of $2.6 \times 10^{-9}$ sbu, bright X-ray knots are generally identifiable with either individual HII regions or larger star-forming complexes chiefly along the spiral arms, as traced by the $\mathrm{H} \alpha$ emission. More interesting is the relationship of the diffuse X-ray emission at lower levels (e.g., $1.3 \times 10^{-9} \mathrm{sbu}$ ) with other components. From the leading edges of the spiral arms toward their downstream sides (e.g., Fig. 6), the dust lanes, together with the $\mathrm{CO}, \mathrm{H} \alpha, \mathrm{FUV}$, and diffuse X-ray emissions, appear in a sequence, representing the dense dusty gas generation, star formation and radiation feedback, and stellar mechanical feedback (see also Jarrett et al. 2013; Poetrodjojo et al. 2019; Koda et al. 2020). Although these components do overlap with each other, their extents increase in the sequence toward the downstreams. Such differential spatial properties, as well as the sub-linear intensity dependence of the X-ray emission on the FUV, reflect on the evolution of the stellar feedback and its coupling with the ISM across the spiral arms into their downstreams.

To further examine the differential properties, we probe how the diffuse soft X-ray emission may be globally traced by the stellar mass (dominated by old stars) and the SFR, as described in $\S 2.2$. Fig. 9 shows the radial intensity distribution $S_{x}(r)$ of the diffuse emission in the $0.45-1 \mathrm{keV}$ band. While the predicted stellar mass contribution is only $\sim 8 \%$, the distribution mimics the shape of the SFR contribution, although a relative shift is present, which will be discussed in $\S 4.2$. We here proceed with a fit with Equation 10 after excluding data points that show the largest deviations, which gives $b=8.5 \times 10^{-13} \mathrm{sbu} /\left(\mathrm{M}_{\odot} \mathrm{yr}^{-1} \operatorname{arcsec}^{-2}\right)$. We consider this fitted (or simply scaled) model as an approximate (or first-order) characterization of the SFR contribution to an accuracy better than $\sim 30 \%$ in the disk region concerned here.

Our main objective here is to facilitate a systematic check of how the observed 2-D diffuse soft X-ray intensity spatially deviates from the SFR distribution. With the above fitted $b$ value, we may now construct a 2-D model distribution of the emission from an adopted SFR distribution $\left(S_{S F R, m}\right)$, together with the stellar mass contribution. (§ 2.2):

$$
S_{x, m}=S_{x, *}+b S_{S F R, m}
$$

We actually build two models. One (Model I) assumes an azimuthally symmetric distribution of the SFR: i.e., $S_{S F R, m}$ in the above equation is represented by the median intensity values in a set of the concentric annuli of the $\Sigma_{S F R}$ image (Equation 9) around the galactic center. This model is used to subtract the contribution (traced by the mean radial diffuse $22 \mu \mathrm{m}$ emission) to the observed $\mathrm{X}$-ray emission, in order to reveal the X-ray enhancements that are associated with SFR peaks, including spiral arms, even when they are in outer disk regions $\left(r \gtrsim r_{c}\right.$; Fig. 10). The FUV peaks, probably except for U-SE, now appear to show enhanced X-ray emission, in contrast to what is seen in Fig. 4B.

To further demonstrate the morphological shifts of the X-ray enhancements relative to the SFR peaks or spiral arms, we construct Model II by considering $S_{S F R, m}$ in Equation 11 to be the values in the $\Sigma_{S F R}$ image (Equation 9). This model assumes that the 2-D SFR distribution spatially traces the X-ray emission. Fig. 11A shows Model II, compared with the observed diffuse soft X-ray intensity contours (same as those in Fig. 4A). By subtracting the model image from the observed one, we obtain the residual image of the X-ray intensity (Fig. 11B). Fig. 11C-D further compare the residual X-ray intensity enhancement contours with the FUV and Spitzer $24 \mu \mathrm{m}$ distributions. The enhancements (e.g., NEE and SWE) appear systematically away from the leading edges of the major spiral arms toward their downstreams and, in particular, tend to fill local valleys in the $24 \mu \mathrm{m}$ intensity distribution. These trends are quantitatively exaggerated here, because Model II simply assumes a linear scaling of the X-ray intensity with the SFR (in contrast to the square-root dependence as indicated in Fig. 8), which leads to over-subtraction (or under-subtraction) in high (or low) SFR regions. Nevertheless, we believe that this result from a simple and relatively independent analysis is qualitatively useful.

Fig. 12 directly compares the azimuthal intensity distributions of the diffuse X-ray intensity (which is calculated with the data without smoothing) and the $24 \mu \mathrm{m}$ emission, which similarly traces the $\mathrm{SFR}$ at a higher resolution than the $22 \mu \mathrm{m}$ image. The relative $\mathrm{X}$-ray enhancements are apparent at $\sim 0^{\circ}-40^{\circ}$ (NEE) and $\sim 180^{\circ}-220^{\circ}$ (SWE) on the downstream sides of the NE and SW arms, respectively.

Furthermore, Fig. 6 shows that the distribution of the diffuse Xray intensity is affected by the presence of dust lanes. This effect is confirmed statistically by the strong anti-correlation between the $\mathrm{X}$-ray intensity and the CI in the spiral arm regions (Fig. 13). The anti-correlation is also apparent in inter-arm regions (Fig. 6).

\subsection{Spectral Properties of the diffuse X-ray emission}

Fig. 14 presents the HR map, providing a model-independent overview of the spectral hardness distribution of the diffuse soft Xray emission across M83. The emission is generally harder along the spiral arms than in their downstream and inter-arm regions. This trend is also illustrated in the positive correlation of the HR with the FUV and X-ray intensities (Fig. 15 A-B). Furthermore, the HR tends to decrease with the increasing radius from the galactic center (Fig. 15C), while locally enhanced along the spiral arms. These trends suggest that the X-ray emission emerging from active starforming regions is both enhanced and hardened.

The hardness should at least partly be due to relatively strong $\mathrm{X}$-ray absorption, especially in the inner part of the galactic disk. Fig. 15C includes the radial CI profile constructed from the HST images. Because of their limited field of view, the CI profile extends only to the off-center radius $r \sim 3^{\prime}$. Nevertheless, the profile shows the flattening of the CI beyond $r \sim 2^{\prime}$, indicating that the reddening does not change much and probably becomes less important in the outer disk field. In contrast, the HR continues to drop from $r \sim 2^{\prime}$ to $r \sim 4^{\prime}$ by $\sim 0.3$. This drop is thus most likely due to the temperature decrease of the plasma with the increasing radius. We speculate that the temperature decrease may be largely due to an adiabatic expansion of the plasma from the inner to outer disk, because the X-ray radiative cooling of the hot plasma is inefficient. However, to calculate the expected HR profile for the adiabatic expansion, one needs to 

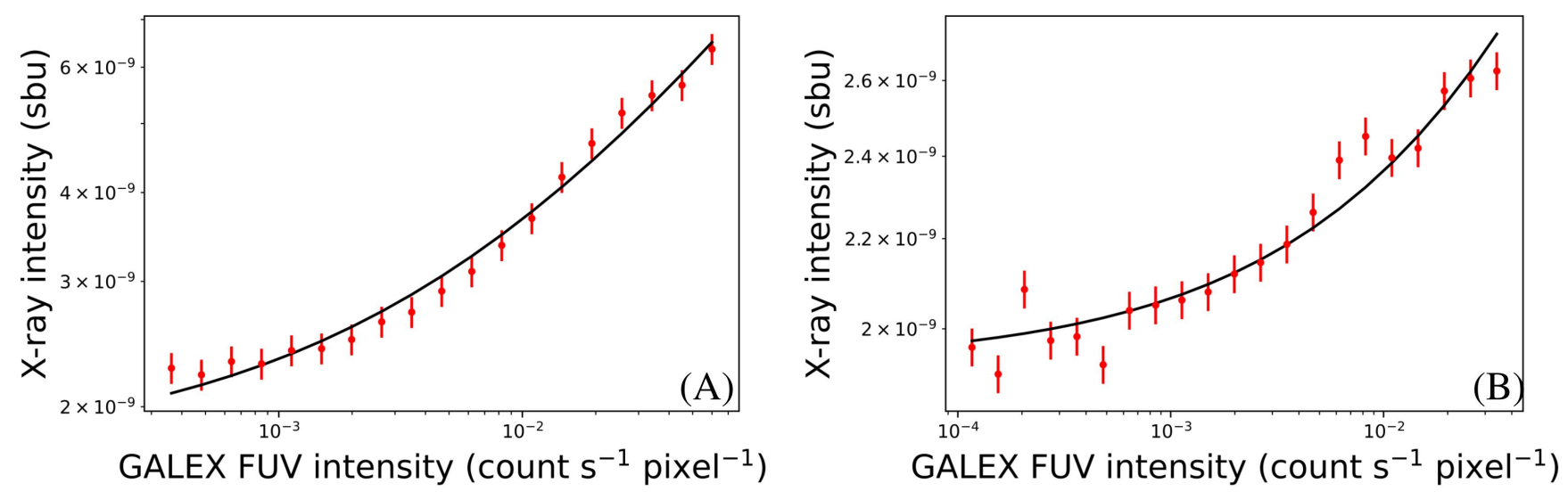

Figure 8. Diffuse soft X-ray intensity vs. GALEX FUV intensity: (A) within $r_{c}$ (but excluding the central region) and (B) between the radius and the $10^{8} \mathrm{~s} c \mathrm{~cm}^{2}$ exposure contour (Fig. 1). The solid curve represents a fit of the data with $S_{x, m}=\left(2.0 \times 10^{-8}\right) I_{F U V}^{1 / 2}+1.7 \times 10^{-9}$ in $\left(\right.$ A) and $S_{x, m}=\left(4.4 \times 10^{-9}\right) I_{F U V}^{1 / 2}+1.9 \times 10^{-9}$ in (B).

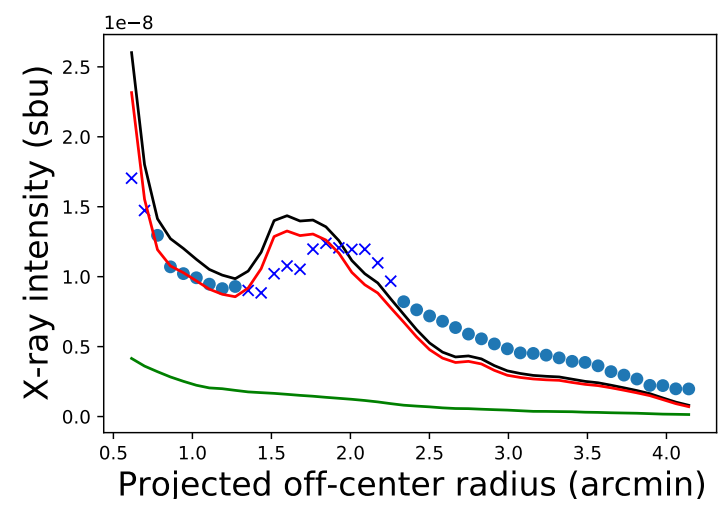

Figure 9. Approximate decomposition of the radial intensity profile of the diffuse $0.45-1 \mathrm{keV}$ emission (blue points) into the contributions due to the stellar mass (green line) and recent SFR (red line). The black line that fits the data

is the sum of the two contributions. This decomposition is based on a model fit (see Equation 10) to the data points marked as circles; those marked as crosses show too large deviations from the model to be included in the fit. The statistical error of individual data points is only $\sim 2 \%$ and is thus not included.

know the position-dependent pressure change of the plasma, which is difficult to infer from the existing data with any certainty.

We characterize the spectral properties of the diffuse X-ray emission from selected individual regions (on- or off-arms), as well as from the inner and outer galactic disks (Fig. 1B). One may expect that the spectral properties in a small specific region are more uniform than over a large field. Thus our characterization starts with the selected on- and off-arm regions.

We first try fits with a simple APEC plasma (plus power law) model,together with the foreground absorption column density $\left(\mathrm{N}_{H}\right)$. Such fits to the on-arm spectra, for example, give the plasma temperatures of $\sim 0.3 \mathrm{keV}$. But the fits with a reduced $\chi_{r}^{2} \gtrsim 2$ are far from being acceptable (e.g., Fig. 16). Allowing the metallicity to vary does not help much to improve the fitting statistic and leads to a value $\lesssim 0.1$ solar, far lower than what is measured for the ISM in M83 and not expected for hot plasma arising from the massive star feedback. Fundamentally, the 1-T plasma model spectrum is too peaked, compared to the data. A plasma of two temperatures (2-T; plus power law) at $\sim 0.2 \mathrm{keV}$ and $\sim 0.7 \mathrm{keV}$ gives an acceptable fit with six fitting parameters: two temperatures and two normalizations, plus $\mathrm{N}_{H}$, and the normalization of the power law, while its index is fixed $(\$ 2)$.

We find that the lognormal plasma plus power law gives a comparably good fit with fewer fitting parameters $(\S 2)$. The quality of the fit and the fitted spectral shape parameters are not particularly sensitive to the metallicity $Z$ as a single parameter (e.g., Fig. 17A), which is thus fixed to the solar value. The normalization or emission measure $(\mathrm{EM})$ of the plasma is nearly proportional to $1 / Z$ because its emission is dominated by metal lines. The best-fit mean temperature is anti-correlated with the dispersion $\sigma_{x}$ and $\mathrm{N}_{H}$ (e.g., Fig. 17B) and is typically not well constrained in a fit, although a one-sided $95 \%$ confidence upper limit is $\sim 0.15 \mathrm{keV}$. To avoid the uncertainty caused by this degeneracy, we simply fix $\bar{T}=0.1 \mathrm{keV}$, consistent with the characterization based on X-ray absorption line measurements of diffuse hot plasma in our Galaxy (e.g., Gissis et al. 2020). This setup of the model leaves $\sigma_{x}$ as the only fitting parameter to characterize the spectral shape of the hot plasma component (e.g., Fig. 18). As described in $\S 2$, further improvements in all the spectra arise from the use of the lognormal absorption model, plus a Galactic foreground fixed at $4.1 \times 10^{20} \mathrm{~cm}^{-2}$. Even in this later modeling, which we finally adopt, the total number of fitting parameters $\left(\sigma_{x}\right.$ and the normalization of the plasma; $\bar{N}_{H}$ and $\sigma_{N_{H}}$ of the absorption; and the normalization of the power law) is one less than the six of the 2-T plasma model mention above.

Table 1 summarizes the results from the fits of the final modeling. We include the fitted or inferred parameters in Table 1. The modeling for the inner and outer disk spectra with excellent counting statistics, in particular, allows for meaningful constraints on key metal abundances, which also give additional improvements of the fits (Fig. 20). The measurements of such individual abundances or their relative values utilize individual spectral line features, which break the above mentioned degeneracy of $Z$ with the normalization of the plasma. The fitted $\mathrm{C}, \mathrm{O}, \mathrm{Ne}, \mathrm{Mg}, \mathrm{Si}$ and $\mathrm{Fe}$ abundances are $4.1_{-4.1}^{+1.0}\left(3.4_{-2.3}^{+1.0}\right), 3.7_{-2.3}^{+0.2}\left(1.6_{-1.2}^{+3.8}\right), 4.9_{-2.9}^{+0.5}\left(2.0_{-1.4}^{+5.0}\right), 3.1_{-1.9}^{+0.4}$ $\left(1.2_{-0.8}^{+2.6}\right), 3.5_{-1.1}^{+0.9}\left(1.0_{-0.9}^{+3.9}\right), 3.1_{-0.9}^{+0.3}\left(0.9_{-0.6}^{+2.3}\right)$ in the inner (outer) disk, respectively. Other $\alpha$ or Fe-like elements are tied to $\mathrm{C}$ or $\mathrm{Fe}$ 

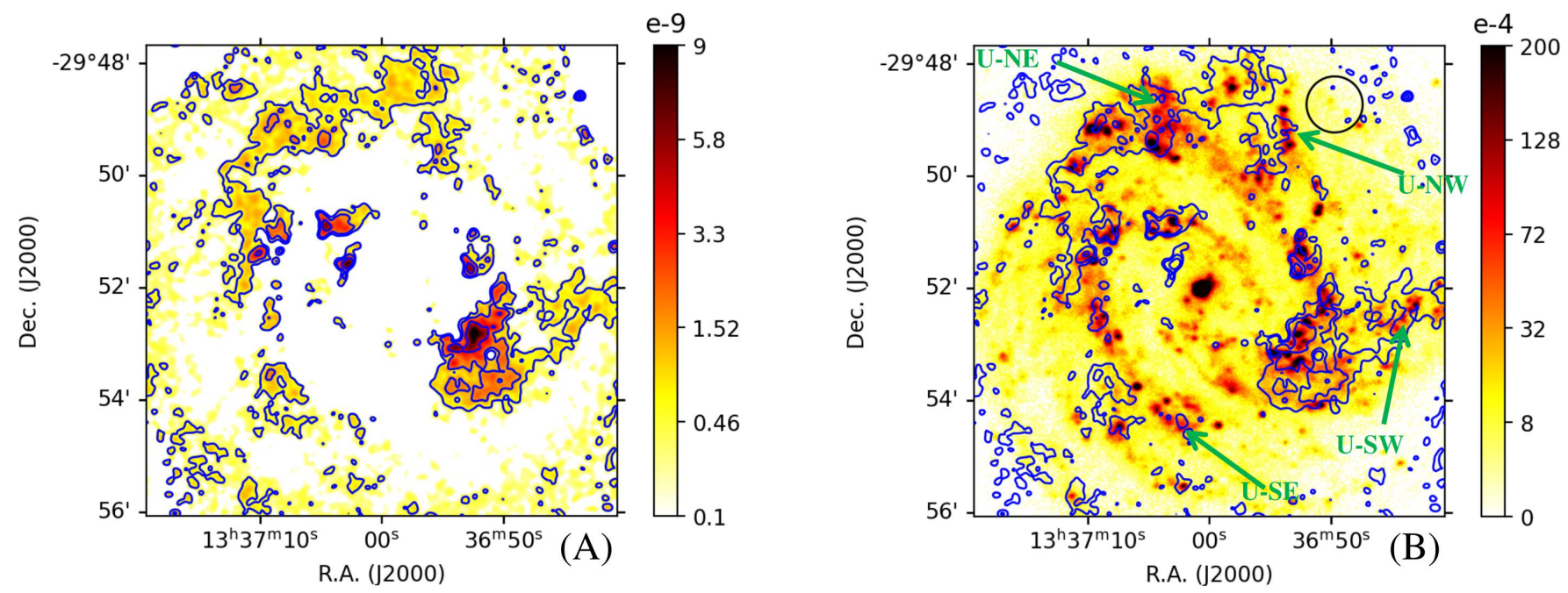

Figure 10. (A) Residual image of the diffuse soft X-ray intensity after the subtraction of the Model I stellar mass and SFR contributions. (B) GALEX FUV image as in Fig. 4. The X-ray intensity contours in both panels are at $(5,15$, and 40$) \times 10^{-10}$ sbu.

in the fits. It should be noted that these absolute values of the abundances are systematically uncertain, since they can be sensitive to our specifically assumed spectral models for the individual components (e.g., the index of the power law: the higher the index, the greater the metal abundances). The $\mathrm{C}$ abundance, in particular, primarily affects the fitting of the low-energy end of the spectra and is thus strongly correlated with the foreground absorption. Considering these statistical and systematical uncertainties, we regard the above fitted abundances as still being consistent with being solar, as has been assumed in the spectral modeling for other regions (Table 1).

Interestingly, the temperature dispersion does not change much from one region to another, all within $\sim 20 \%$ of the mean value $\sigma_{x} \sim 0.65$. Hence no clear evidence is seen here for the cooling of the plasma from the on-arm to inter-arm regions, for example. This is somewhat surprising, as one may expect that the heating of the plasma by CC SNe should be highly concentrated in spiral arms. The measurement of $\sigma_{x}$ may be complicated by uncertainties in the spectral separation between the lognormal plasma and power law components, as well as the degeneracy with $\bar{N}_{H}$ and $\sigma_{N_{H}}$. These latter two parameters are also strongly anti-correlated with each other (Table 1). It is thus important to consider both parameters in determining the role of the absorption in shaping the observed X-ray spectra.

The spectral fitting results shows that the soft X-ray emission in M83 is dominated by the thermal plasma. The power law to plasma flux ratio $\left(f_{p l} / f_{t h}\right)$ in the $0.5-2 \mathrm{keV}$ band is $\lesssim 9 \%$. The flux of the power law component is consistent with the combined contribution from the spill-over of the excised discrete sources and discrete sources that are below our detection limit, including faint HMXBs and young stellar objects as traced by the SFR (e.g., Bogdán \& Gilfanov 2011; Mineo et al. 2012). The power law contribution becomes even smaller in the $0.45-1 \mathrm{keV}$ band $(\lesssim 5 \%)$, which we use to map the diffuse soft X-ray intensity distribution.

Using the $0.5-2 \mathrm{keV}$ luminosity of the lognormal plasma of the inner (outer) disk, together with SFR $=1.4(0.5) \mathrm{M}_{\odot} \mathrm{yr}^{-1}$ in the same region, we estimate $R_{X, S F R}=L_{0.5-2 \mathrm{keV}} / S F R=5.2(4.9) \times$ $10^{39} \mathrm{erg} \mathrm{s}^{-1} /\left(\mathrm{M}_{\odot} \mathrm{yr}^{-1}\right)$, respectively.

With the fitting results, we infer various physical parameters of the plasma, assuming an isobaric state (Cheng et al. 2021). Our spectral fitting, however, is independent of this assumption, which affects only the interpretation of the fitted parameters and the subsequent inferences. The thermal pressure $\left(P_{t h}\right)$ can then be expressed as

$$
\begin{aligned}
P_{t h} & =\sqrt{\frac{4 \pi D^{2} \eta^{2} K_{t h}}{10^{-14} V_{t}}}\left(k_{B} \bar{T}\right) e^{\sigma_{x}^{2}} \\
& \approx\left(1.0 \times 10^{33} \mathrm{keV} \mathrm{cm}^{-3}\right) \sqrt{\frac{K_{t h}}{V_{t}}}\left(k_{B} \bar{T}\right)_{k e V} e^{\sigma_{x}^{2}},
\end{aligned}
$$

where $\eta \approx 2.1$ and the distance to M83 are adopted, while $K_{t h}$ is the normalization of the lognormal plasma component (Table 1). The effective total volume $\left(V_{t}\right)$ of the hot plasma is $V_{t}=f_{h} V$, where $f_{h}$ is the effective volume filling factor of the plasma in a physical volume $(V)$. For a face-on galaxy like M83, we may approximate $V$ as the area of each region (as projected in the sky) multiplied by the depth $h \approx 1 \mathrm{kpc}$ along the line of sight: i.e., $V \approx\left(1.4 \times 10^{61} \mathrm{~cm}^{3}\right) A h_{k p c}$, where $A$ (in units of $\operatorname{arcsec}^{2}$, as given in Table 1) is the area from which the spectrum is extracted. The total thermal plasma energy is simply $E_{t h}=\frac{3}{2} P_{t h} V_{t}$, while the integrated EM and mass of the plasma can be expressed as

$$
E M=\left[\frac{P_{t h}}{\eta k_{B} \bar{T}}\right]^{2} V_{t} e^{-2 \sigma_{x}^{2}},
$$

and

$$
M_{t h}=\frac{P_{t h} \mu m_{p} V_{t}}{k_{B} \bar{T}} e^{-\sigma_{x}^{2} / 2},
$$

where $\mu$ is the mean molecular weight of particles (electrons and ions) and $m_{p}$ is the proton mass.

While the numerical results of these estimations for our spectral analysis regions are included in Table 1 , the above three equations show how $P_{t h}, E M$ and $M_{t h}$ analytically depend on $\sigma_{x}$, as well as on those parameters common to the 1-T plasma (e.g., $\bar{T}$ and $K_{t h}$ ). $P_{t h}$ in the lognormal plasma is a factor of $e^{\sigma_{x}^{2}}$ greater than in an isothermal state (i.e. $\sigma_{x}=0$ ) with a uniform density and at $\bar{T}$. This factor corrects for the bias introduced by the EM weighting of the temperature: a large fraction of the volume, occupied by the plasma at the higher temperature (and hence lower density) side of the lognormal distribution, does not contribute much to the observed soft Xray emission. Similarly, assuming the uniformity and the single $P_{t h}$ would over-estimate $E M$ and $M_{t h}$ of the lognormal plasma, which 


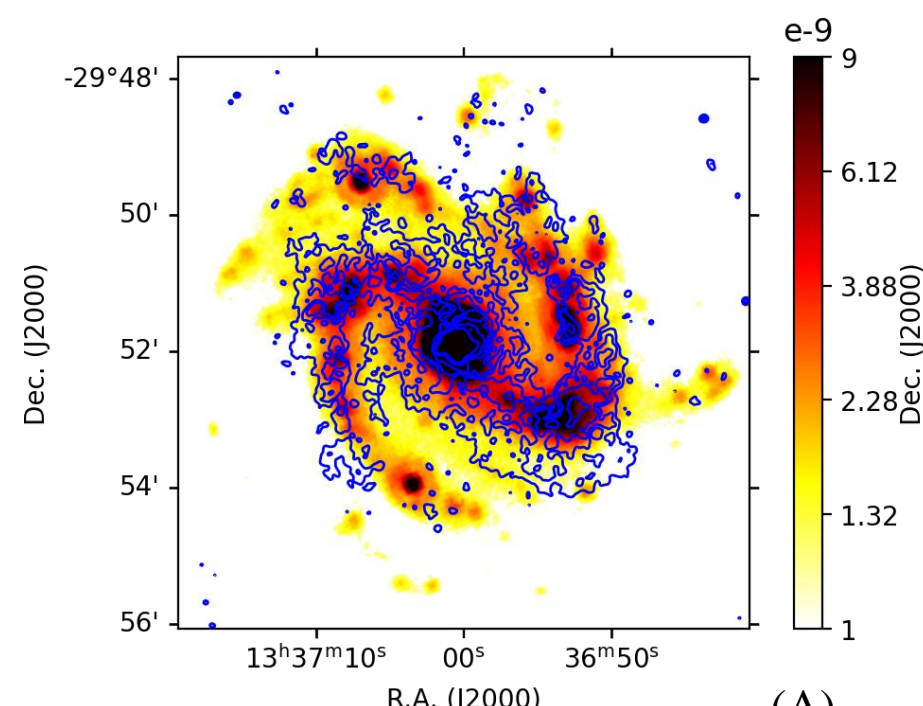

(A)
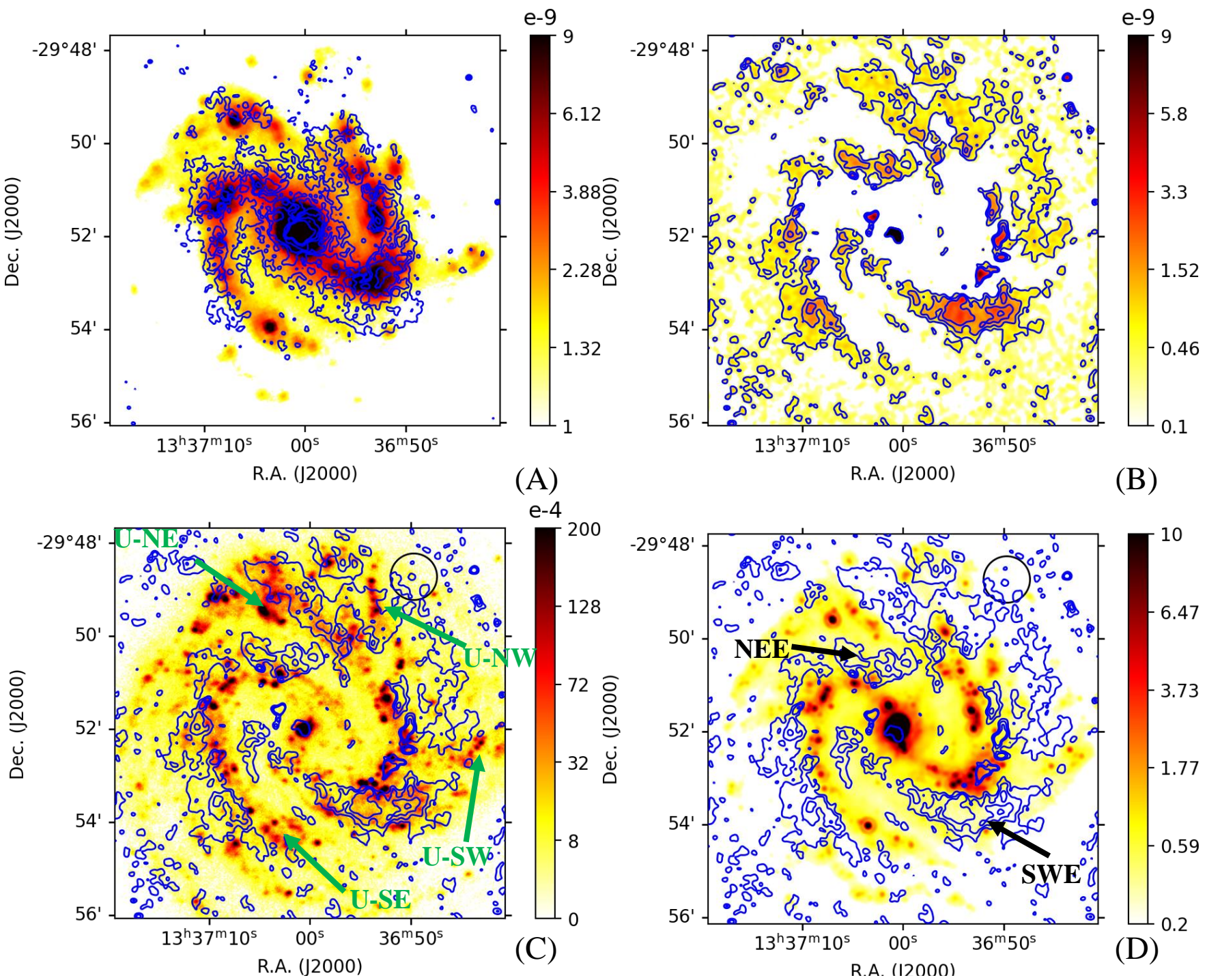

Figure 11. (A) The same contour as in Fig. 4, but overlaid on the Model II prediction of the combined stellar mass and SFR contributions. (B) The residual image of the diffuse soft X-ray data minus the model II prediction in the intensity range of $(1-90) \times 10^{-10}$ sbu with an overlaid representative residual intensity contours at $5 \times 10^{-10}, 1.2 \times 10^{-9}$, and $2 \times 10^{-9} \mathrm{sbu}$. (C) These same contours overlaid on the FUV image as in Fig. 4. (D) The same contours overlaid on the $24 \mu \mathrm{m}$ intensity image, together with the (green) $22 \mu \mathrm{m}$ contours at $(0.5,1$ and 2$) \times\left(4.3 \mathrm{DN} \mathrm{s}^{-1} \operatorname{arcsec}^{-2}\right)$ for comparison (Fig. 7). Marked are two large-scale $\mathrm{X}$-ray intensity excesses in the downstreams of the spiral arms: the northeast excess (NEE) and the southwest excess (SWE), which can also be appreciated in the azimuthal intensity distribution presented in Fig. 12.

results in the correction factors of $e^{-2 \sigma_{x}^{2}}$ and $e^{-\sigma_{x}^{2} / 2}$ of their respective expressions.

It should be noted that $\bar{T}$ itself does not necessarily define the observed X-ray spectral shape or even its peak location. In our spectral modeling, much of the X-ray emission from the plasma at the lower temperature side of $\bar{T} \sim 0.1 \mathrm{keV}$ is absorbed by cool gas along the line-of-sight. The observed X-ray arises mostly from plasma at an intermediate temperature (typically $\sim 0.2-0.7 \mathrm{keV}$, as may be characterized by a simple (1- or 2-T) plasma fit (e.g., Owen \& Warwick 2009; Kuntz \& Snowden 2010). For example, such a temperature appears at $\sim 1.5 \sigma$ above $\bar{T}=0.1 \mathrm{keV}$ for the lognormal plasma in the inner disk (Table 1): i.e., $T=\bar{T} e^{1.5 \sigma_{x}}=0.32 \mathrm{keV}$. The corresponding density at this temperature is a factor of 3.2 lower than at $\bar{T}$. These dependencies demonstrate the importance of considering the plasma temperature distribution in determining the plasma properties.

We present in Appendix $C$ the mapping of the spectral parameters
(Fig. C1A-C), based on the lognormal plasma plus power law model. Both $\sigma_{x}$ and plasma normalization maps show a NE-SW elongation and are generally correlated with the spiral arms. The morphological similarity of these two maps is at least partly due to the high degeneracy between the two parameters in spectral fits. The power law normalization map reveals a linear structure northeast of the galactic center. This NW-SE oriented structure overlaps with the FR II radio galaxy, but seems to extend further to the SE, reaching the eastern spiral arm in projection. It is not clear, however, as to whether or not the entire structure is coherent one or if it is all due to the radio galaxy. There are other apparent peaks in the power law normalization map, which look quite faint, round and extended. They could represent clustered background AGN, which are not detected individually. Although our adopted simple spectral model may be a good approximation globally (due to the averaging), it may not be appropriate on small scales (e.g., due to stochastic spectral realization of 
Table 1. Spectral fitting results

\begin{tabular}{|c|c|c|c|c|c|c|c|c|c|c|c|c|c|c|}
\hline Regions & $\bar{N}_{H}$ & $\sigma_{N_{H}}$ & $\sigma_{x}$ & $K_{t h}$ & $K_{p l}$ & $\chi^{2} / \mathrm{dof}$ & Area & $f_{t h}$ & $f_{p l}$ & $L_{t h, x}$ & $P_{t h}$ & $E_{t h}$ & $E M$ & $M_{t h}$ \\
\hline On-arm Region I & $26_{-5}^{+11}$ & $0.04_{-0.03}^{+0.20}$ & $0.54_{-0.05}^{+0.05}$ & $6.3_{-24}^{+1.4}$ & $5.8_{-21}^{+2.1}$ & $70 / 63$ & 8195 & 1.3 & 0.05 & 5.2 & 0.032 & 9.0 & 1.5 & 1.6 \\
\hline On-arm Region II & $7_{-1}^{+18}$ & $0.21_{-0.06}^{+0.40}$ & $0.64_{-0.07}^{+0.08}$ & $2.8_{-1.6}^{+0.4}$ & $8.2_{-2.0}^{+2.2}$ & $89 / 75$ & 8009 & 1.3 & 0.09 & 2.6 & 0.025 & 6.8 & 1.1 & 1.2 \\
\hline Off-arm Region & $6_{-1}^{+-1}$ & $\begin{array}{l}-0.06 \\
0.28_{-0.28}^{+0.20}\end{array}$ & $\begin{array}{l}-0.07 \\
0.54_{-0.04}^{+0.04}\end{array}$ & $\begin{array}{l}-1.6 \\
1.7_{-0 .}^{+0.2}\end{array}$ & $\begin{array}{l}-2.0 \\
1.1_{-0.6}^{+0.7}\end{array}$ & $90 / 72$ & 4740 & 0.42 & 0.01 & 1.4 & 0.022 & 3.6 & 0.4 & 0.7 \\
\hline Inner Disk & $6_{-6}^{+12}$ & $0.18_{-0.13}^{+0.24}$ & $0.75_{-0.03}^{+0.04}$ & $7.3_{-0.6}^{+5.3}$ & $59_{-5}^{+5^{\circ}}$ & $553 / 508$ & 88669 & 9.7 & 0.8 & 17 & 0.014 & 42 & 1.7 & 7.4 \\
\hline Outer Disk & $10_{-9}^{+6}$ & $0.08_{-0.01}^{+0.01}$ & $0.79_{-0.09}^{+0.11}$ & $3.3_{-1.4}^{+6.5}$ & $8.6_{-5.9}^{+5.7}$ & $538 / 508$ & 133820 & 3.5 & 0.12 & 5.2 & 0.008 & 37 & 0.8 & 3.8 \\
\hline
\end{tabular}

Note: Listed parameters are obtained from the best-fit spectral model consisting of the lognormal plasma and a power law, plus the lognormal absorption, as well as a foreground absorption of a column density fixed to the Galactic value of $N_{H}=4.1 \times 10^{20} \mathrm{~cm}^{-2}$. The mean temperature $\left(k_{B} \bar{T}\right)$ is fixed to $0.1 \mathrm{keV}$. The metal abundances are fixed to the solar values, except for the inner and outer disk regions, for which key elements are fitted (see $\S 3.2$ for the results). $\bar{N}_{H}$ is the mean column density of intrinsic absorbing gas in units of $10^{20} \mathrm{~cm}^{-2}$, while $\sigma_{N_{H}}$ is the dispersion of the column density in logarithm (Cheng et al. 2021). $\sigma_{x}$ is the dispersion of the temperature in logarithm, while $K_{t h}$ and $K_{p l}$ are the normalizations of the lognormal plasma and power law components in units of $10^{-3} \mathrm{~cm}^{-5}$ and $10^{-6}$ photons $\mathrm{cm}^{-2} \mathrm{~s}^{-1} \mathrm{keV}^{-1}$ at $1 \mathrm{keV}$ (Cheng et al. 2021). $\chi^{2} / \mathrm{dof}$ are the $\chi^{2}$ value divided by the degree of freedom (dof) of each fit. Also given are the areas (in units of $\operatorname{arcsec}^{2}$ ) from which the spectra are extracted, as well as derived parameters: the 0.5-2 keV absorbed (no absorption correction) fluxes of the thermal and power law components $\left(f_{t h}\right.$ and $f_{p l}$ in units of $10^{-13} \mathrm{erg} \mathrm{cm}^{-2} \mathrm{~s}^{-1}$ ), the (unabsorbed) $0.1-10 \mathrm{keV}$ luminosity $L_{t h, x}\left(10^{39} \mathrm{erg} \mathrm{s}^{-1}\right.$ ), thermal pressure $P_{t h}\left[\left(f_{h} h_{\mathrm{kpc}}\right)^{-1 / 2} \mathrm{keV} \mathrm{cm}^{-3}\right]$, energy $E_{t h}\left[10^{54}\left(f_{h} h_{\mathrm{kpc}}\right)^{1 / 2} \mathrm{erg}\right]$, emission measure $E M\left(10^{63} \mathrm{~cm}^{3}\right)$ and mass $M_{t h}\left(10^{7} \mathrm{M}_{\odot}\right)$ of the plasma.

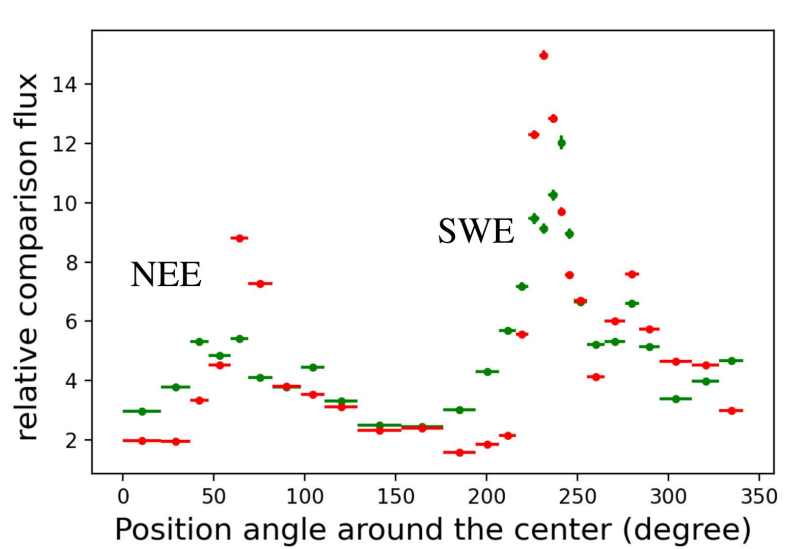

Figure 12. Comparison between the azimuthal intensity distribution of the diffuse $0.45-1 \mathrm{keV}$ intensity (represent by the green points) and the Spitzer $24 \mu \mathrm{m}$ (red points) emissions in the $(0.5-0.8) r_{c}$ annulus around the center of M83. The position angle (x-axis) is in an easterly direction from north. Marked are the two large-scale X-ray intensity excesses, NEE and SWE (see also Fig. 11).

a few faint discrete sources, affecting the power law, or local hot plasma or enhanced X-ray absorption, altering the spectral shape in the soft band). With these in the consideration, we find that the fits are generally acceptable across the galaxy (Fig. C1D), although individual features in the the maps need to be treated with caution.

To check how the spectral properties may be affected by the Xray absorption traced by the optical CI, we present in Fig. 21 three spectra extracted from regions of different CI ranges. Guided by the general trend that the HR increases with the $\mathrm{CI}$, we extract the spectra from the regions with $C I<1.2\left(\mathrm{CI}_{1}\right), 1.2<C I<1.75\left(\mathrm{CI}_{2}\right)$, and $C I>1.75\left(\mathrm{CI}_{3}\right)$. For ease of comparison, these three spectra have been scaled to the same effective extraction area of $10^{4} \operatorname{arcsec}^{2}$. The $0.5-1 \mathrm{keV}$ fluxes in $\mathrm{CI}_{2}$ and $\mathrm{CI}_{3}$ are only $79 \%$ and $52 \%$ of the flux in $\mathrm{CI}_{1}$, whereas the $1-4 \mathrm{keV}$ fluxes are consistent with each other. A straight-forward interpretation of these soft X-ray flux deficiencies is that the X-ray absorption is enhanced with the increasing reddening, as traced by the CI. We further conduct a joint fit to the three spectra with the lognormal plasma plus power law model, in which

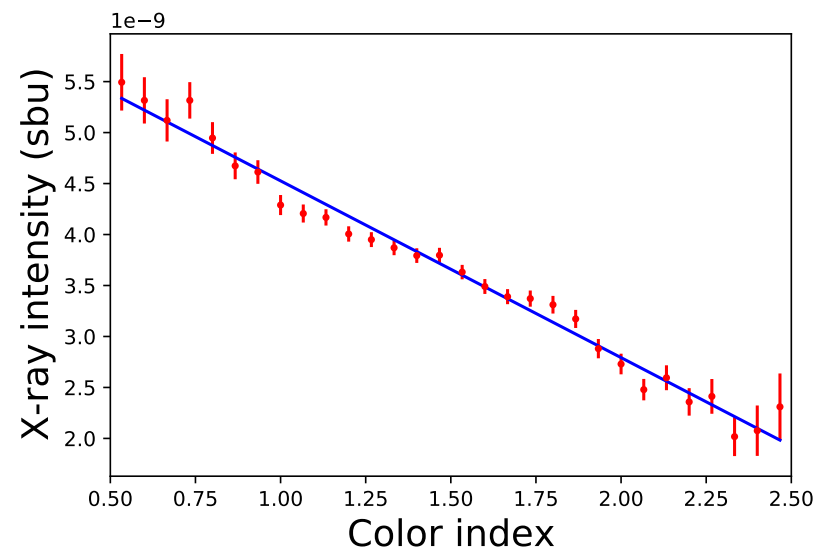

Figure 13. Diffuse $0.45-1.0 \mathrm{keV}$ intensity as a function of the $\mathrm{CI}$ in the two spiral arm regions (Fig. 1B). The solid line is a linear fit with the slope of $-1.7 \times 10^{-9}$ sbu per CI.

the $\bar{T}, \sigma_{x}$ and $\Gamma$ are linked to avoid the large estimation uncertainties of these intrinsic spectral shape parameters which would otherwise be introduced by the degeneracy among them. For the same reason, we use a single $N_{H}$ to characterize the foreground absorption of each spectrum. However, the quality of this joint fit is poor (judged by its $\chi^{2} /$ dof. So the fitted parameters, especially the absolute $N_{H}$ values for the individual spectra, may not be quite meaningful, physically. The real situation for the X-ray emission and absorption configuration can be considerably more complicated than what is assumed in this spectral modeling (see more discussion on this in $\S 4.4$ ). Nevertheless, the qualitative trend of the X-ray absorption increasing with the $\mathrm{CI}$ is quite convincing.

\section{DISCUSSION}

We here synthesize the above results and explore their implications. We start with a comparison of this study on M83 with existing ones, especially the one on M101 (KS10), and then discuss major issues related to the production and evolution of diffuse hot plasma, as well 


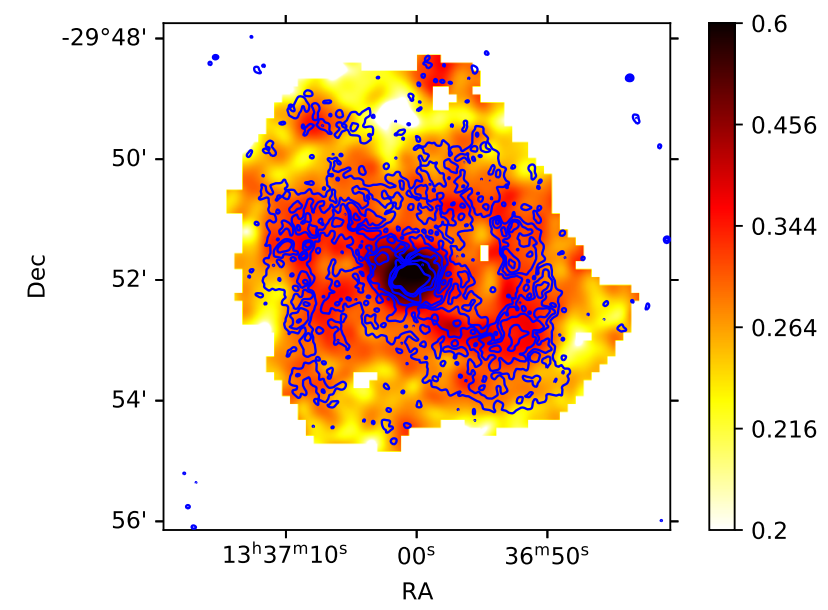

Figure 14. HR $[I(0.7-1.0 \mathrm{keV}) / I(0.45-0.7 \mathrm{keV})]$ map created from the intensity images smoothed with a Gaussian of $\sigma=7$ ". 87 . Only regions where HR $S / N>3$ are presented here. The overlaid intensity contours are the same as that in Fig. 4.

as its spatial and thermal structure, and to the X-ray absorption effect of dusty gas in M83. These discussions provide useful insights into the coupling of the stellar feedback with various phases of the ISM.

\subsection{Comparison with the existing studies on M101}

This comparison is aimed to enhance our understanding of the results on both M83 and M101. M101 is a prototypical ScI galaxy at the distance of $6.8 \mathrm{Mpc}$. The work by KS10 is based on similar deep Chandra ACIS observations. Various trends observed in M101 are generally consistent with our results on M83 presented above. We find that the contribution from its old stellar population is small and that the diffuse soft X-ray intensity is strongly correlated with the surface SFR on M83, consistent with the conclusion reached on M101 (Figs. 4B and 8), showing a similar square-root proportionality. Qualitatively, this sub-linear proportionality is not difficult to understand (e.g., KS10). The FUV emission peaks early in the evolution of a massive star cluster (before the first $\mathrm{SN}$ ), while the X-ray luminosity of the surrounding hot plasma represents the prolonged heating of the ISM by the mechanical energy feedback, which includes both fast stellar winds and $\mathrm{SNe}$ and accumulates with time. The observed diffuse soft X-ray intensity is further affected by the foreground absorption, as well as the evolution of the plasma (e.g., expansion, mass-loading, and potentially cooling). This convolution of the stellar evolution and feedback with the plasma heating and evolution, together with the foreground extinction/absorption, tends to produce a shallow X-ray intensity dependence on the FUV emission. The square-root dependence, demonstrated here and in KS10, can thus be used as a quantitative constraint on theory and/or simulation for the stellar feedback/ISM coupling.

Also consistent between the M101 and M83 studies is the apparent weakness of the diffuse X-ray emission from some moderately bright FUV peaks in relatively outer galactic disk regions. As shown in $\S 3.1$, part of this weakness is due to the underlying lower diffuse X-ray background emission in such outer regions. Another contributing factor to the X-ray weakness is expected from the generally decreasing metallicity of the ISM with the off-center radius. According to Hernandez et al. (2019), the metallicity of M83 is $Z \approx+0.20$ dex above the solar value at the galactic center, continuously declines with the radius, and then sharply breaks down to a rather flat distribution at $-0.20 \operatorname{dex}$ at $R / R_{25} \approx 0.4-0.5$, where $R_{25}=6.44$ is the isophotal radius of the galaxy. Interestingly, the break radius occurs roughly at $r_{c}$. This decline and drop of the metallicity decreases the plasma emissivity (by a factor of $\sim 2$ ). In addition, the density and pressure of the ISM on average decreases with the radius (e.g., Table 1), leading to lower plasma emission measure in superbubbles (Mac Low \& McCray 1988). These factors together should largely explain the relative weak X-ray emission from recently star-forming regions in the outer galactic disk.

M83 and M101 do exhibit interesting differences. While the former contains a distinct bar, which seems to be responsible for the two grand-design spiral arms of the galaxy. In contrast, M101 shows swirling spiral arms, which are highly asymmetrical and multi-facet and probably result from the galaxy's interaction with neighboring dwarf galaxies. This interaction may also account for the asymmetric appearance of M101: The western half of the galaxy appears considerably dimmer than the eastern part in both FUV and X-ray (KS10); Giant H II regions (NGC 5461, NGC 5462, and NGC 5471) are all present in an extended spiral arm on the eastern part of the galaxy. These exceptionally bright regions show high HR in the range of $\sim 0.6-1.1$. There are no such regions in M83, the star formation of which is more smoothly distributed along its two grand spiral arms. The surface SFR is greatly enhanced in the circumnuclear region of the galaxy, compared to that of M101. These differences between the two galaxies largely explain their different HR dependence on the off-center radius. Fig. 15A exhibits a sharp HR increase (from $\sim 0.55$ to $\gtrsim 1)$ from the off-center radius $r \sim 1^{\prime}$ to the nucleus of M83. A similar plot for M101 (Fig. 8 in KS10) shows a rather flat distribution of the HR $(\sim 0.4)$, reaching $\sim 0.55$ at the very nuclear region.

In both M101 and M83, X-ray emission in general appears more extended than the FUV light. This is likely due to the longer cooling time scale of diffuse hot plasma than the lifetime of $\mathrm{O}$ and $\mathrm{B}$ stars that are largely responsible for the FUV emission. However, the confusion among close segments of successive spiral arms along the flow lines prevents a quantitative estimate of the X-ray cooling times for this galaxy. In contrast, the association of the diffuse X-ray emission with the two prominent spiral arms is more distinct in M83, which makes it an excellent site to study the structure and evolution of hot plasma due to the stellar feedback.

\subsection{Structure of the downstream diffuse X-ray emission in M83}

An important result of our study is the detection of the enhanced diffuse X-ray emission on the downstream side of the spiral arms. There are multiple reasons for the lack of the detection of the downstream enhancement in previous Chandra studies (e.g., Tyler et al. 2004). Almost all of them are based on data that are not sufficiently deep and/or are very much based visual examination of the $\mathrm{X}$-ray emission with various tracers of recent star formation and/or gas/dust associated with spiral arms. It is also possible that the relationship of diffuse X-ray emission with star forming regions depends on the dynamics and/or trigger mechanism of spiral arms, which may vary from one galaxy to another (e.g., see the relevant discussion on M101 and M83 in $\S 4.1$ and Shabani et al. 2018). As described in $\S 1$, in recent years no much attention has been placed to the global ISM, especially its hot component, on scales of spiral arms, both theoretically and observationally. Detailed studies on such scales will be essential to the understanding of the stellar feedback and ISM coupling.

The diffuse soft X-ray enhancement observed in the downstreams of the spiral arms in M83 appears to be consistent with a scenario of 

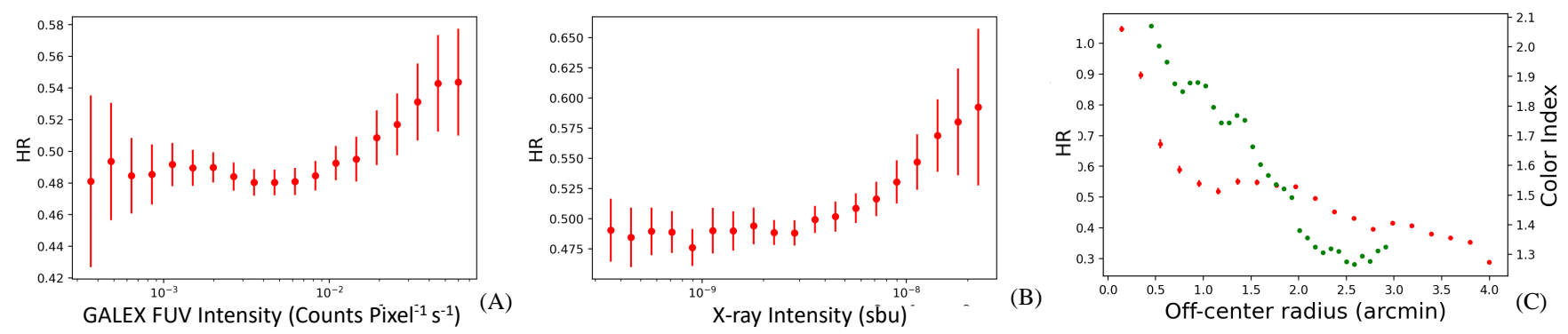

Figure 15. HR as the function of the GALEX FUV intensity (A), the diffuse $0.45-1 \mathrm{keV}$ intensity (B), and the off-center radius (C). For comparison, Panel (C) also includes the radial distribution of the CI (green).

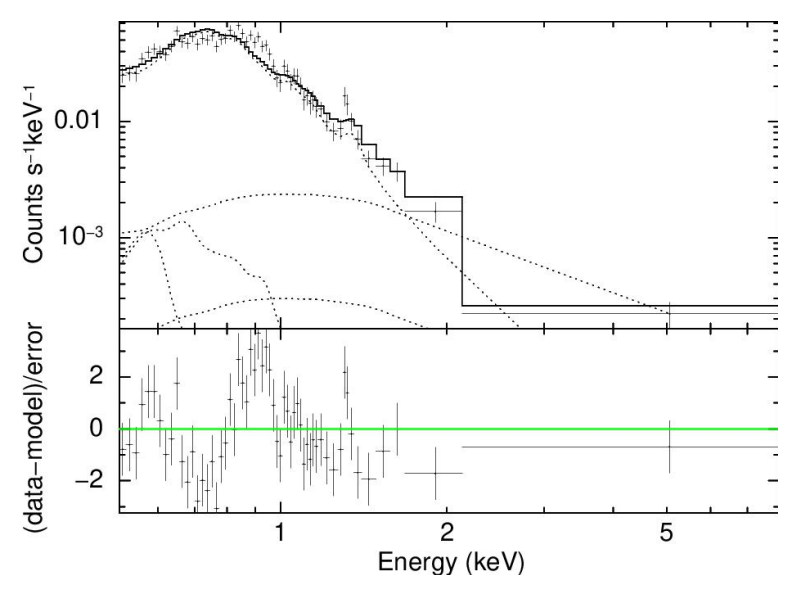

Figure 16. ACIS-S3 spectrum extracted from On-arm Region I (Fig. 1B), fitted with a single-temperature plasma (APEC) plus a power law. Individual components (including those associated with the local X-ray background) are separately plotted as dotted lines; the APEC and power law components are seen as the top two lines at $\sim 1 \mathrm{keV}$. This model is an inadequate characterization of the data.

ISM heating by stellar mechanical feedback. In the downstreams, superbubbles produced by individual massive stellar clusters may have merged to form supergiant bubbles or tunnels of hot plasma on $\mathrm{kpc}$ scales comparable to the size of individual spiral arms. Along the way, massive stars are ending their lives and their clusters are being dissolved (e.g., Lada \& Lada 2003; Portegies Zwart et al. 2010). In this scenario, the overall width of the X-ray enhancement associated with each spiral arm is related to the motion of massive stars relative to the spiral arm pattern. Indeed, the enhancement is largely observed within $r_{c}$. M83 has a flat rotation curve at $180 \mathrm{~km} \mathrm{~s}^{-1}$ and a spiral arm pattern speed of $50 \mathrm{~km} \mathrm{~s}^{-1} \mathrm{kpc}^{-1}$ Lundgren et al. (2004). So at a representative radius of $\sim 3 \mathrm{kpc}$ (where the rotation period is $\sim 1.0 \times 10^{8} \mathrm{yrs}$ ), for example, the rotation speed of the stars relative to a spiral arm may be estimated as $\sim 30 \mathrm{~km} \mathrm{~s}^{-1}$. Assuming a standard initial mass function of massive stars, their mechanical energy input rate (chiefly via CC-SNe) remains nearly constant over $\sim 3.2 \times 10^{7} \mathrm{yrs}-$ the lifetime of a $8 M_{\odot}$ zero-age main sequence star (e.g., Leitherer et al. 1999). During this time period, such a star will move away from the spiral arm with an offset of $\sim 19^{\circ}$, which is comparable to the mean value observed for the enhancement. The overall extent of the enhancement is expected to be larger, depending on the time scale of the plasma cooling, expanding, and/or escaping into the galactic halo. Therefore, the association of diffuse soft X-ray emission with the downstreams is, at least qualitatively, expected from the heating of the ISM by energetic feedback from massive stars formed in spiral arms.

The expansion of the hot plasma in and around the galactic disk is consistent with the observed radial distribution trends of the diffuse soft X-ray emission ( $(3)$. While the outward shift of the emission relative to the star formation regions (Fig. 9) can be a direct consequence of the plasma expansion on the galaxy scale, the decreasing HR with the off-center radius (Fig. 15C) is expected from the cooling of the plasma, even just adiabatically.

Incidentally, the same stellar feedback scenario may explain phenomena observed in other wavelengths. The cosmic-ray generation accompanied by the plasma heating may naturally account for the presence of warm molecular gas, as indicated by the elevated $\mathrm{CO}$ $\mathrm{J}=2-1 / 1-0$ ratio observed in the same downstreams (Koda et al. 2020). Magnetic field configuration in the ISM can also be shaped by the feedback. Significant polarized radio emission is detected in the inter-arm regions (Weżgowiec et al. 2020), which are away from the downstreams where the enhanced diffuse X-ray emission is observed. This is consistent with the hypothesis that the generation of the hot plasma is largely responsible for the turbulence, which tends to wipe out organized magnetic fields in the ISM (e.g., Bacchini et al. 2020).

\subsection{Validity and implication of the lognormal models of the plasma and absorption}

With a continuous temperature distribution, the lognormal plasma characterization should be more physical than those involving two or more discrete temperature components (Cheng et al. 2021; Vijayan \& Li 2021). The lognormal distribution is expected from the central limit theorem for a variable (e.g., temperature) characterizing the accumulation of many independent multiplicative random processes such as repeated shock-heating of the plasma, as demonstrated in various hydrodynamic simulations (e.g., Frank et al. 2014). Of course, a continuous temperature distribution is also expected from plasma cooling, radiatively or via turbulent mixing (e.g. Lancaster et al. 2021). In any case, the lognormal plasma characterization includes or extends the single-temperature model with only one additional parameter (the dispersion of the temperature in logarithm). In comparison, the modeling with plasma of multiple temperatures would add more parameters, which typically have little actual physical meaning and are forced by the discrete setup (e.g., Gayley 2014; Vijayan \& Li 2021).

Similarly, the lognormal characterization of the X-ray absorption (LNABS) should also be more realistic than the assumption of a single foreground absorbing gas column density, as commonly used. 

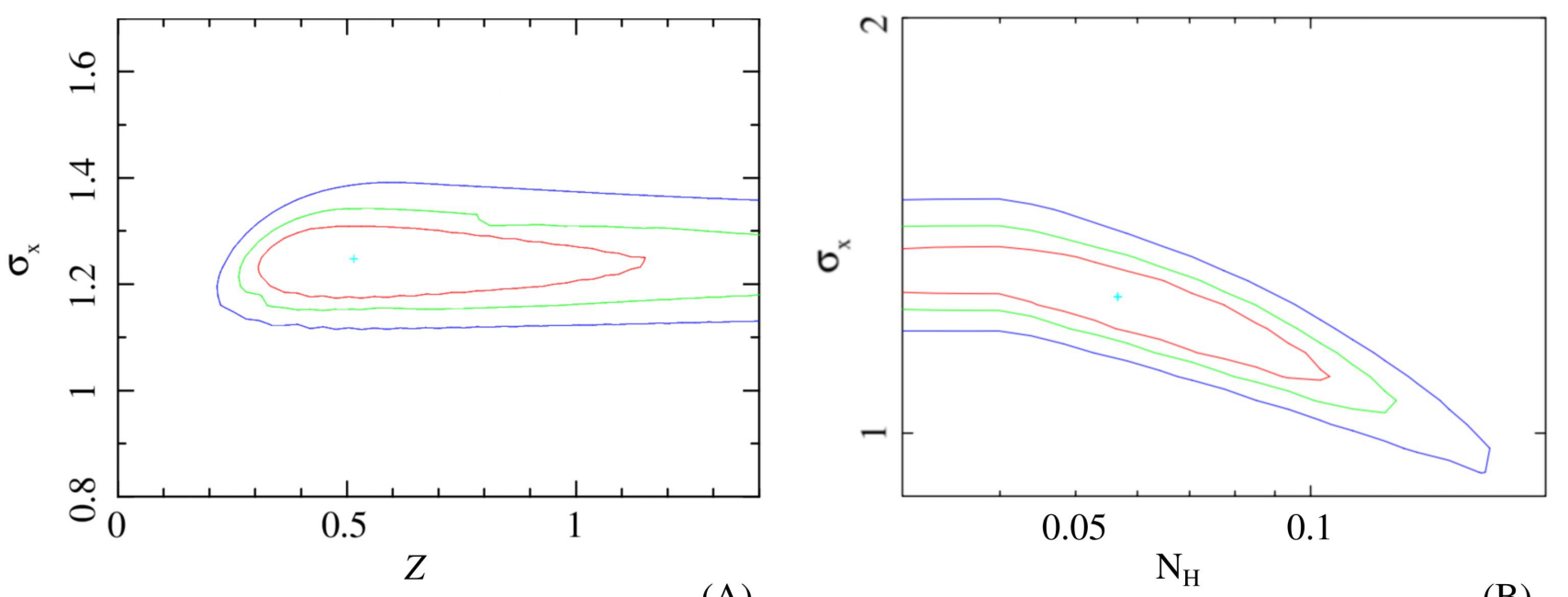

(A)

(B)

Figure 17. Illustration of the a parameter degeneracy in a spectral fit: the lognormal temperature dispersion $\left(\sigma_{x}\right)$ vs. the metallicity ( $Z$ in units of the solar value) of the plasma (A) or the foreground X-ray-absorbing gas column density $\left(N_{H}\right.$ in units of $10^{22} \mathrm{~cm}^{-2}$; B). The contours are at the 68,90 , and $99 \%$ confidences around the best-fit parameters (marked by the plus sign) for On-arm Region I.
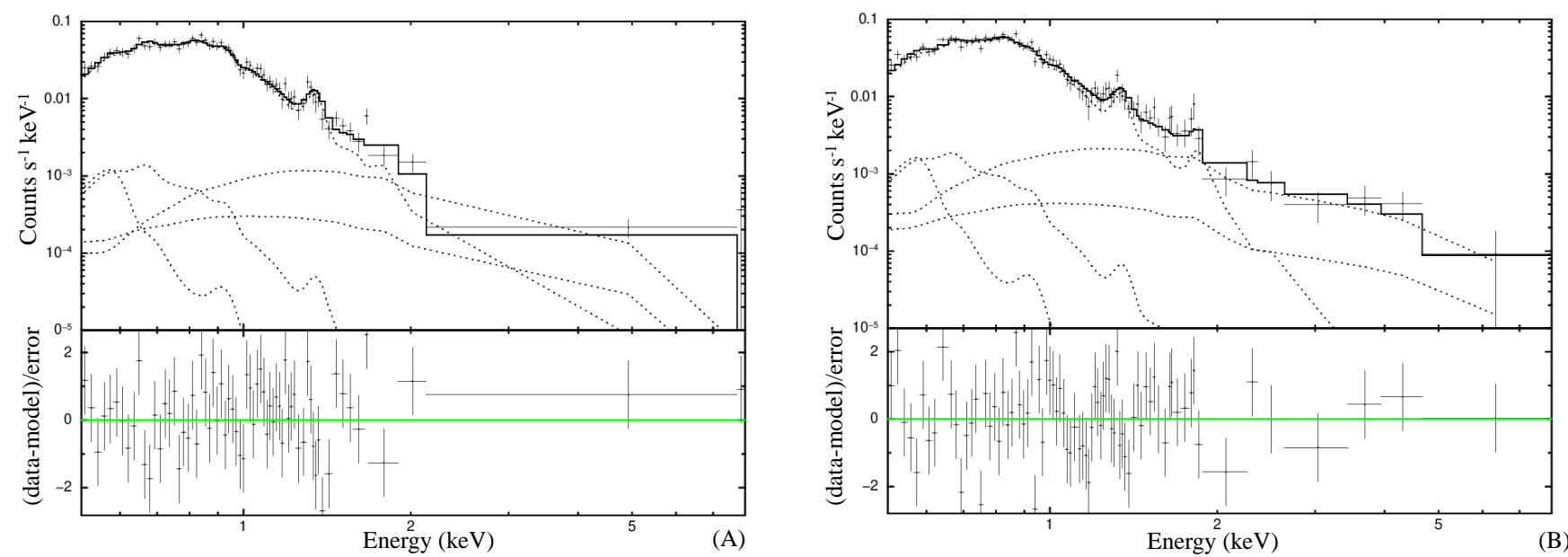

Figure 18. ACIS-S3 spectra of On-arm Regions I (A) and II (B), fitted with the lognormal plasma plus power law model (Table 1). Individual components (including those associated with the local X-ray background) are separately plotted as dotted lines; the on-arm lognormal plasma and power law components are seen as the top two lines at $\sim 2 \mathrm{keV}$.

Strong X-ray absorption variation is clearly seen across individual star forming regions (e.g., Cheng et al. 2021). Such variation is expected to be greater across much of a star forming galaxy, including both on- and off-arm regions.

Indeed, as we have demonstrated in $\S 3.2$, our spectral analysis shows the effectiveness of the lognormal characterizations of the plasma temperature and absorbing gas column density distributions. In particular, no evidence exists for a preferred peak in the temperature range that the $\mathrm{X}$-ray data are sensitive to $(\gtrsim 0.1 \mathrm{keV})$. The single temperature plasma fit is not acceptable when the $\mathrm{S} / \mathrm{N}$ of a spectrum is good (e.g., Fig. 16). The lognormal plasma with the mean temperature fixed to $0.1 \mathrm{keV}$ (thus the same number of fitting parameters as the 1-T model) gives much improved fits to all the spectra that we have analyzed. The application of the lognormal absorption, instead of a single absorption, further improves the fits (\$ 3.2). Our results also show that the combined applications of the lognormal plasma and absorption models lead to the estimates of the metal abundances that are consistent with or slightly higher than the metallicity measured from the integrated UV light of star clusters ( $\$ 4.1$; Hernandez et al. 2019). In contrast, X-ray spectral fits with plasma of discrete temperatures tend to give estimates of the metal abundances that are substantially lower than measured values for ISM or young stars (e.g., Wang et al. 2001; Kuntz \& Snowden 2010; Anderson et al. 2016; Bogdán et al. 2017), which is inconsistent with the stellar feedback enrichment scenario.

It is useful to compare our results here with those on the 30 Doradus nebula - the starburst region in the Large Magellanic Cloud. Cheng et al. (2021) have found that the spectrum of the diffuse X-ray emission from the nebula can also be well modeled with the lognormal plasma, although its mean temperature is found to be $0.3 \mathrm{keV}$, significantly higher than the upper limit found here for the spectra extracted over the relatively large regions in M83 (Table 1). The 


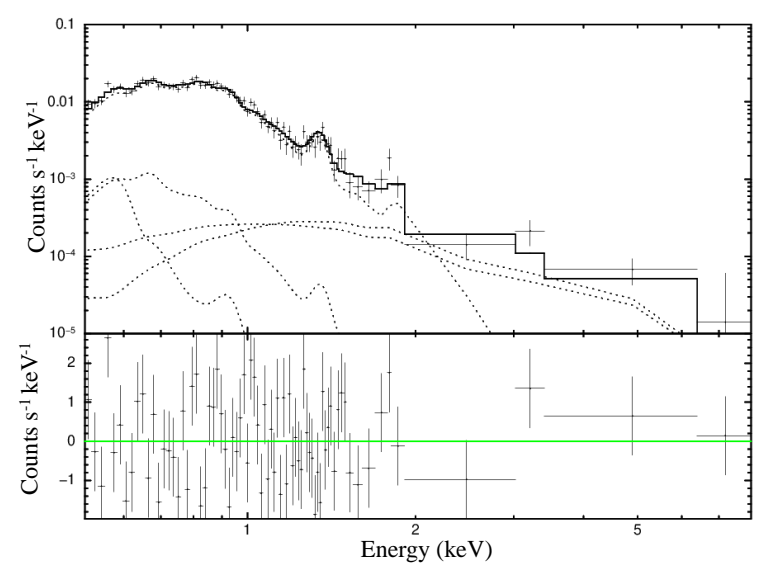

Figure 19. The same as Fig. 18, but for a representative off-arm region.

lower mean temperature of the plasma in the M83 may be partly due to the spectral inclusion of multiple plasma components over vast physical regions. Even the spectra of our on-arm regions contain the large-scale diffuse X-ray emission contributions in the galactic disk and corona, because only the off-galaxy background is subtracted. Therefore, the effective application of the same modeling for M83 demonstrates that the lognormal plasma may be used to effectively account for the temperature distribution across large physical regions.

We further explore limitations and uncertainties of the lognormal plasma modeling. Even under the simple one-zone (single uniform pressure) isobaric plasma assumption, the interpretation of the physical parameters (e.g., $P_{t h}$ ) is not trivial. As shown in $\S 3.2$, their inferences depend on the validity of the lognormal plasma modeling over a broad temperature range - an issue that needs to be carefully examined. Theoretically, it remains unclear as to how well a lognormal plasma may describe the spectrum of diffuse X-ray emission from a complex system like a galaxy. Existing studies based on computer simulations are still too limited to allow for a quantitative comparison with our results. Nevertheless, studies such as those conducted by Vijayan \& Li (2021) are useful for finding qualitative trends. Their SFR10 simulation of a galaxy with a Milky-Way-like mass and a $\mathrm{SFR}=10 \mathrm{M}_{\odot} \mathrm{yr}^{-1}$, for example, shows that the hot circumgalactic medium exhibits a general correlation of $T \propto 1 / n$ over the $10^{5}$ to $10^{7} \mathrm{~K}$ range, as expected in an isobaric state. But around this correlation is also a large dispersion, which may be expected for hot plasma with a range of thermal pressure and is not accounted for in our simple lognormal plasma modeling. It is thus highly desirable to properly model such diversity in the density and temperature distribution, as well as their intrinsic correlation. In addition, the uncertain parameters of $h_{k p c}$ and $f_{h}$ also need to be characterized before one can effectively use our derived parameters with confidence.

In any case, the distribution of the temperature down to $\lesssim 0.1 \mathrm{keV}$ can have strong implications for the radiative cooling of the plasma. We may estimate the $X$-ray cooling time scale of the plasma as $t_{c}=E_{t h} / L_{t h, x}$. The parameters in Table 1 indicate that $t_{c}$ is in the range of $(6-24) \times 10^{7}\left(f_{h} h_{\mathrm{kpc}}\right)^{1 / 2}$ yrs, shorter in the on-arm regions and longer in the outer disk field. This time scale likely represents an upper limit to the total cooling time scale of the plasma. In an isobaric state, for example, the time scale is $\propto T^{2}$. For plasma at temperatures smaller than $0.1 \mathrm{keV}$, the radiation is released mostly in the very soft X-ray to extreme UV range and is hence absorbed by cool interstellar gas. Such low-temperature plasma may partly result from mixing layers between hot plasma and cool gas (e.g., Begelman
\& Fabian 1990; Fielding et al. 2020; Lancaster et al. 2021). Therefore, heat transfer and radiative cooling may, in principle, consume much of the stellar mechanical feedback energy within the galactic disk of M83.

\subsection{Structure of the X-ray absorbing gas}

According to the results presented in $\S 3$, the absorption seems to play a major role in determining the observed morphology, intensity, and spectrum of the diffuse soft X-ray emission. The effectiveness of the absorption is intimately related to the actual line-of-sight distribution of the cool ISM relative hot plasma, which is largely unknown.

Dust lanes are visible across much of the galaxy's surface (Fig. 6), not only at the leading edges of the majestic spiral arms, but also in regions between them, forming "spurs" or "feathers". They may represent clouds that rapidly grew, were then differentially compressed in spiral arms and have later been shear-stretched in inter-arm regions (Shetty \& Ostriker 2006). Alternatively, such clouds may represent inflow streams under the bar potential and/or outflows from the galactic disk (e/g., Kannan et al. 2021; Farber \& Gronke 2021). In any case, such dust lanes, partly traced by ${ }^{12} \mathrm{CO}$ emission, are likely interspersed with $\mathrm{H} \alpha$ - and diffuse X-ray-emitting plasma. While ${ }^{12} \mathrm{CO}$ can often be found right at dust lanes, they tend to be anti-correlated with the diffuse $\mathrm{H} \alpha$ and X-ray emissions. Take the outstanding SWE as an example. It is positionally coincident with a region that shows relatively strong diffuse $\mathrm{H} \alpha$ emission and little optical reddening. The X-ray intensity becomes dimmer systematically with increasing reddening or $\mathrm{CI}$ in the region toward the east into inner regions of the galaxy; Fig. 6). This trend is also confirmed in the spiral arm regions, where a linear anti-correlation between the diffuse soft X-ray intensity and the CI is observed (Fig. 13). Assuming that the X-ray absorption is foreground, an observed spectrum is $S_{\varepsilon}=S_{\varepsilon, 0} e^{-N_{H} \sigma_{\varepsilon}}$, where $S_{\varepsilon, 0}$ and $\sigma_{\varepsilon}$ are the X-ray emission spectrum and absorption cross-section as the functions of the photon energy $(\varepsilon)$ and $N_{H} \propto$ CI. Thus a linear anti-correlation between the $\mathrm{X}$-ray intensity and CI may be expected only when the absorption is small (i.e., $N_{H} \sigma_{\varepsilon} \ll 0$ ). However, this condition is apparently inconsistent with the large $\mathrm{X}$-ray intensity variation range that is more than a factor of 2. At least two complications are not accounted for here. Firstly, our measured X-ray intensity is over a broad band so that the X-ray absorption also depends on the spectral shape of the $\mathrm{X}$-ray emission, which remains quite uncertain. Secondly and probably more importantly, the dusty gas is likely inter-mixed with Xray-emitting plasma or not completely foreground; only the emission beyond the gas is subject to the absorption. The net effect of these complications, though difficult to quantify, should naturally lead to the flattening of the X-ray absorption dependence on the CI: e.g., from the nearly exponential form to the observed linear anticorrelation.

The intermixing scenario of the X-ray emission and absorption is consistent with existing observations. For example, Liu et al. (2013) show that the optical extinction in M83 is dominated by dust in a foreground screen, while Mosenkov et al. (2018) estimate that the average face-on optical V-band depth of such dusty screen is $\sim 1$ for a sample of galaxies. The spectral energy distribution modeling of the dust emission suggests that the dusty gas is probably strongly inhomogeneous and clumpy, although its heating mechanism remains largely uncertain and the line-of-sight projection makes it hard to discern the structure. The anti-correlation of the diffuse soft X-ray intensity with the $\mathrm{CI}$, as detected in the present study, indicates that the responsible dusty gas is mostly on the near side of the hot plasma, 

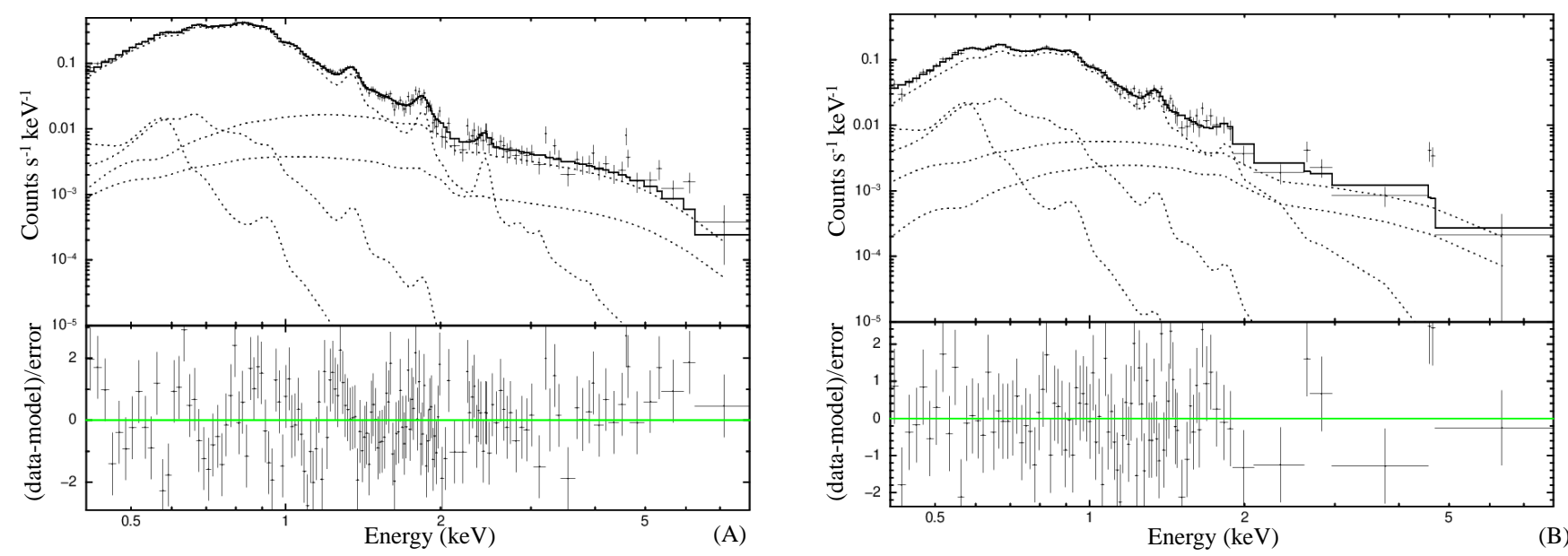

Figure 20. The same as Fig. 18, but for (A) the inner disk spectrum extracted from the region within $r_{c}$, excluding the central region; (B) the outer disk spectrum extracted from the region between $r_{c}$ and the outer exposure contour of $10^{8} \mathrm{~s} \mathrm{~cm}^{2}$ (Fig. 1).

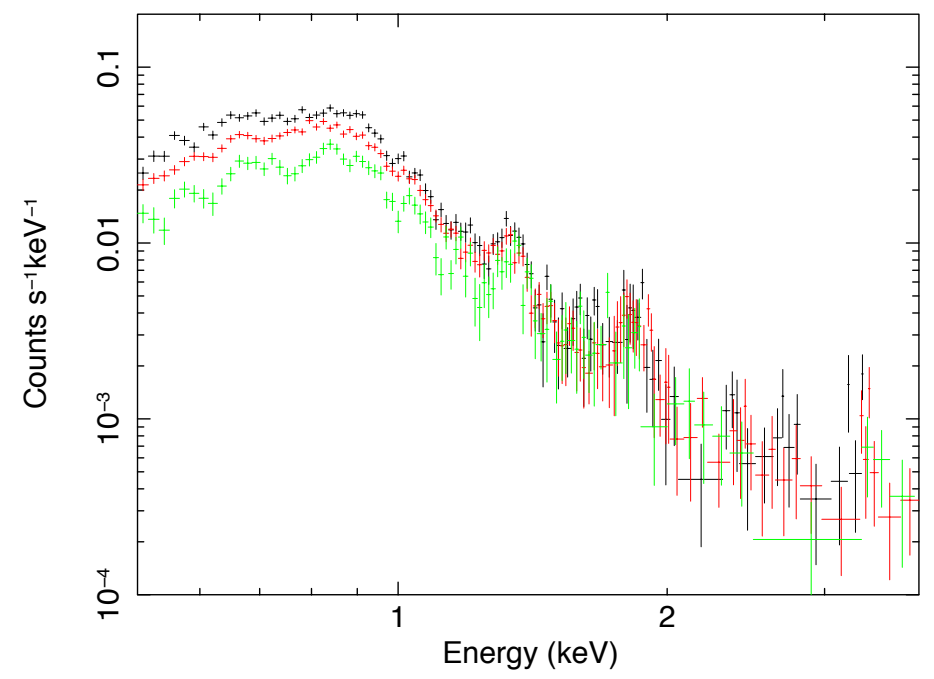

Figure 21. Comparison of the three spectra extracted in the regions with $\mathrm{CI}<1.2$ (black), between $1.2<\mathrm{CI}<1.75$ (red), or $\mathrm{CI}>1.75$ (green).

which is likely located primarily in a thick disk of a characteristic vertical exponential height of $\sim 1 \mathrm{kpc}$ (Boettcher et al. 2017; Nakashima et al. 2018; Mosenkov et al. 2018; Jiang et al. 2019). This scale should be a factor of a few greater than the scale height of the stellar light. Therefore, the dusty gas should lie mainly away from the galactic plane and likely represent blown-out supershell walls interacting with a relatively slow rotating gaseous coronae of M83, probably similar to so-called intermediate velocity or even high-velocity dusty clouds or complexes (Marasco et al. 2012). This interaction could lead to the observed lagging halo of cool gas (e.g., Peek et al. 2009; Röhser et al. 2016) and to the condensation of the coronal gas (Armillotta et al. 2016; Vijayan et al. 2020; Hobbs \& Feldmann 2020; Dutta et al. 2021; Gronke et al. 2021). A systematic study of the interaction, confronted by the observed X-ray absorption properties, will thus help to understand the circulation of the cool dusty gas and plasma at galactic disk/halo interfaces (e.g., Armillotta et al. 2016; van de Voort et al. 2021).

\subsection{Plasma energetics}

We have measured the X-ray luminosity of the diffuse thermal plasma, which can be compared with the stellar mechanical energy input in M83. A useful comparison is the specific luminosity $R_{X, S F R} \approx 5 \times 10^{39} \mathrm{erg} \mathrm{s}^{-1} /\left(\mathrm{M}_{\odot} \mathrm{yr}^{-1}\right)$, as we have estimated from the thermal spectral component in $\S 3.2$. This value is consistent with that estimated for M83 based on XMM-Newton observations (Owen \& Warwick 2009).

To further probe the link between the luminosity of the diffuse soft $\mathrm{X}$-ray emission and the stellar mechanical energy input, we estimate the CC-SN rate from the SFR within $r_{c}$ of M83. Using the local background-subtracted WISE $22 \mu \mathrm{m}$ image, the integrated SFR is $\sim 1 \mathrm{M}_{\odot} \mathrm{yr}^{-1}$ in the M83 disk region or a factor of $\sim 2.3$ higher if the the central $\left(43^{\prime \prime}\right.$ radius) region is included. The corresponding energy input rate in the disk is then $3 \times 10^{41} \mathrm{erg} \mathrm{s}^{-1}$, assuming the conversion of $\sim 1 \mathrm{SN}$ per $100 \mathrm{yr}$ per $1 \mathrm{M}_{\odot} \mathrm{yr}^{-1}$ (Botticella et al. 2012) and $10^{51}$ erg energy per SN. Therefore, the luminosity of the soft X-ray emission within the disk $\sim 1.7 \times 10^{40} \mathrm{erg} \mathrm{s}^{-1}$ (Table 1) implies that the X-ray emission efficiency of the CC-SN mechanical energy feedback is $\sim 10 \%$, which likely represents the lower limit to the bolometric radiation fraction of the energy input. At present, however, the bolometric luminosity or the radiative cooling rate of the plasma cannot be reliably estimated, because of the very uncertain extinction/absorption correction ( $\$ 4.3)$.

Dedicated simulations could be helpful in determining the interplay between the stellar feedback and the ISM. Indeed, existing simulations of active star-forming disk galaxies already show that a large fraction $(\gtrsim 0.2)$ of the mechanical energy released by SNe could vent into the galactic halo powering a strong galactic wind. This fraction depends on the condition for star cluster-driven superbubbles to break out of galactic disks, which corresponds to a surface SFR threshold (e.g. Fielding et al. 2018; Kim et al. 2020) $\sim 0.03 \mathrm{M}_{\odot} \mathrm{yr}^{-1} \mathrm{kpc}^{-2}$, equivalent to $I_{22}=4.3 \mathrm{DN} \mathrm{s}^{-1} \operatorname{arcsec}^{-2}$ (Equation 9). Figs. 7 and 11 show that this threshold can be readily met in a good fraction of the spiral arms in M83. The blowout of hot plasma from these most energetic regions can lead to the local reduction of the X-ray enhancement (due to the EM decrease) or the 
sub-linear X-ray intensity dependence on the FUV, to the redistribution of chemically-enriched hot plasma (in the downstreams and to larger galactic radii), and to the heating of the galactic corona, responsible for the galaxy-wide diffuse emission as has been studied with the XMM-Newton data (Owen \& Warwick 2009).

It remains unclear as to how the mechanical energy input from massive stars is eventually released from a galaxy like M83. According to Mac Low \& McCray (1988), about half of the input energy should initially be in hot gas, while the rest is lost in the formation and kinetic energy of the cool supershells around the superbubbles. However, we have shown that the X-ray luminosity from the galactic disk of M83 is $\sim 10 \%$ of the energy input. Studies of edge-on disk galaxies with various SFRs have further concluded that galactic coronae can account for only a comparable fraction of the energy input (e.g., Li \& Wang 2013). One possibility is that superbubbles are effective acceleration sites of cosmic rays (e.g. Lingenfelter 2019; Cheng et al. 2021), which can escape into the galactic halo and beyond without much noticeable X-ray radiation. Another possibility is that M83 has a strong galactic wind, which drives the bulk of the hot plasma energy out of the immediate vicinity of the galaxy to regions beyond a few tens of $\mathrm{kpc}$. It is also possible that much of the energy in the hot plasma is transformed to cool gas and/or dust (e.g., via turbulent mixing) and is then radiated in other wavelengths (e.g., extreme UV and IR; Begelman \& Fabian e.g., 1990; Fielding et al. e.g., 2020; Lancaster et al. e.g., 2021). In any case, the Xray luminosity limits observed in galactic disks and coronae provide fundamental constraints on any theory about the coupling between the stellar feedback and the ISM.

High desirable are new dedicated simulations, including spiral arm dynamics, to confront the results presented here. They need to have sufficiently high spatial and time resolutions to allow for examination of diffuse hot plasma flows and their interplay with other ISM components, addressing such questions as: How does the hot plasma evolve, dynamically, chemically, and thermally? What is its temperature distribution? How is it mixed with X-ray-absorbing cool gas? The simulations will then enable direct confrontation with the data presented here to test our understanding of the astrophysics underlying the coupling between the feedback and the ISM.

\section{SUMMARY AND CONCLUSIONS}

We have conducted a detailed analysis of diffuse soft X-ray emission in the field of M83, including two outstanding extended background sources. A background FR II radio galaxy and its radio lobes are found to be associated with part of a linear hard X-ray feature. A strongly extended emission peak is attributed to a cluster of galaxies at $z \sim 0.5$ and with an X-ray luminosity of $\sim 3.1 \times 10^{43} \mathrm{erg} \mathrm{s}^{-1}$. The X-ray absorption inferred from the spectrum of this cluster is consistent with the estimate based on $\mathrm{HI}$ and $\mathrm{CO}$ measurements in the galactic disk of M83. The focus of our study is, however, chiefly on spatial and thermal properties of the diffuse X-ray emission in the disk, especially in and around its two grand spiral arms, and on gaining insights into the coupling of the stellar feedback with the ISM. Our main results and conclusions are as follows:

(i) The enhancement of the diffuse X-ray emission is strongly correlated with the spiral arms, is bordered by dust lanes on the upstream side of the spiral arms, and is interspersed with ${ }^{12} \mathrm{CO}$ emission peaks, as well as $\mathrm{H} \alpha$ and FUV emissions. Therefore, the enhancement is closely connected to the feedback from massive stars and may be globally characterized by the ratio $L(0.5-$ $2 \mathrm{keV}) / \mathrm{SFR}=5 \times 10^{39} \mathrm{erg} \mathrm{s}^{-1} /\left(\mathrm{M}_{\odot} \mathrm{yr}^{-1}\right)$. (ii) We confirm the square-root dependence of the X-ray enhancement on the FUV intensity, which is first observed in M101. This sub-linear dependence can be understood as the convolution of the FUV emission evolution of massive stars with the heating, expansion, outflow, and potentially cooling of the diffuse hot plasma.

(iii) Essentially all relatively bright features or peaks of the FUV emission show enhanced diffuse emission in the $0.45-1 \mathrm{keV}$ band. This enhancement tends to decrease with increasing distance from the galactic center, which can be qualitatively explained by the decreasing metallicity and density/pressure of the ISM toward the outer disk of the galaxy.

(iv) We reveal enhanced X-ray emission in the downstreams of the spiral arms. This enhancement tends to fill those so-called interarm fork/void regions of the galactic disk and apparently represents the prolonged mechanical feedback from massive stars over a lifetime of $\sim 32 \mathrm{Myr}$, coupled with the cooling and/or escaping time of the diffuse hot plasma in the downstreams.

(v) The HR ( $\left.=S_{0.45-0.7} / S_{0.7-1.0}\right)$ is positively correlated with the diffuse X-ray intensity and generally decreases with the increasing galactic radius in the disk. Part of these trends is due to the soft X-ray absorption, which increases in star-forming regions, especially in the inner disk of the galaxy. But cooling may also play an important role, although the thermal X-ray luminosity of the plasma accounts for only $\sim 10 \%$ of the mechanical energy inputs from SNe.

(vi) The spectra of the X-ray emission can be reasonably well characterized by an optically-thin thermal plasma with a lognormal temperature distribution of its emission measure. There is no spectral evidence for discrete temperature components. The mean temperature of the lognormal plasma $\bar{T} \lesssim 0.15 \mathrm{keV}$ indicates that much of its radiation occurs in the photon energy range $\lesssim 0.5 \mathrm{keV}$ and is not well traced by the X-ray observations. The X-ray spectral shape of the plasma emission can be well characterized by the lognormal temperature dispersion alone, while $\bar{T}=0.1 \mathrm{keV}$ is fixed. The metal abundances of the plasma are estimated to be generally super solar, especially in the inner galactic disk region within the co-rotation radius, and tend to be higher than the metallicity measurements based on the integrated UV light of star clusters, although a consistency cannot be completely ruled out because of systematic uncertainties in our X-ray spectral modeling.

(vii) The differential X-ray absorption inside M83 plays an important role in determining both the spectral shape and the intensity distribution of the observed diffuse soft X-ray emission. The effect of the differential absorption on the X-ray spectra can be reasonably well characterized by a lognormal distribution of the absorbing-gas column density. The surface intensity of the emission is overall anticorrelated with the dust reddening in an approximately linear fashion.

(viii) The X-ray emission and absorption are most likely intermixed globally, which may represent outflows of multi-phase gas from recent massive star-forming regions. Indeed, a good fraction of them, as judged by their surface SFR, seem to be sufficiently energetic to produce such outflows. The geometry of the inter-mixing remains largely unknown, however, which prevents a reliable estimation of the X-ray absorption effect and hence the radiative cooling rate of the diffuse hot plasma in the galactic disk.

(ix) The bulk of the mechanical energy feedback from massive stars is not released in the soft X-ray. Nevertheless, the X-ray measurements provide fundamental constraints on any theory for the feedback/ISM coupling. Possible additional channels for the feedback energy release include the cosmic-ray, galactic wind, and/or cooling of the hot plasma via mixing with cool gas/dust and radiating at longer wavelengths. It will be important to directly confront 
the X-ray measurements with dedicated simulations, including the spiral arm dynamics, and to probe the astrophysics of the coupling between the stellar feedback and the ISM.

\section{ACKNOWLEDGMENTS}

We thank the referee for constructive comments, which helped to improve the paper, appreciate the help of Yingjie Chen in the implementation of the VLNTD and LNABS models, and are grateful to Tom Jarrett for sharing the WISE $22 \mu \mathrm{m}$ and radio continuum images of M83 used in our multi-wavelength comparison and to Brian Alden for sharing his ClusterPyXT routine, which helped in our construction of the spectral maps. The research of D.Q. Wang and Y.X. Zeng at University of Massachusetts was partly supported by NASA grants, while Ákos Bogdán acknowledges support from the Smithsonian Institution.

\section{DATA AVAILABILITY}

The X-ray data on M83 as described in $\S 2$ include all Chandra ACIS-S observations taken on the galaxy before 2020 and are available in the Chandra data archive (https://asc.harvard.edu/cda/). Processed data products underlying this article will be shared on reasonable request to the authors.

\section{REFERENCES}

Afruni A., Fraternali F., Pezzulli G., 2021, MNRAS, 501, 5575

Alden B., Hallman E. J., Rapetti D., Burns J. O., Datta A., 2019, Astronomy and Computing, 27, 147

Anderson M. E., Churazov E., Bregman J. N., 2016, MNRAS, 455, 227

Armillotta L., Fraternali F., Marinacci F., 2016, MNRAS, 462, 4157

Bacchini C., Fraternali F., Iorio G., Pezzulli G., Marasco A., Nipoti C., 2020, A\&A, 641, A70

Begelman M. C., Fabian A. C., 1990, MNRAS, 244, 26P

Bell E. F., de Jong R. S., 2001, ApJ, 550, 212

Boettcher E., Gallagher J. S. I., Zweibel E. G., 2017, ApJ, 845, 155

Bogdán Á., Gilfanov M., 2011, MNRAS, 418, 1901

Bogdán Á., Bourdin H., Forman W. R., Kraft R. P., Vogelsberger M., Hernquist L., Springel V., 2017, ApJ, 850, 98

Botticella M. T., Smartt S. J., Kennicutt R. C., Cappellaro E., Sereno M., Lee J. C., 2012, A\&A, 537, A132

Brown M. J. I., Jarrett T. H., Cluver M. E., 2014, Publ. Astron. Soc. Australia, 31, e049

Cheng Y., Wang Q. D., Lim S., 2021, MNRAS, 504, 1627

Cowan J. J., Roberts D. A., Branch D., 1994, ApJ, 434, 128

Dale D. A., et al., 2009, ApJ, 703, 517

Dame T. M., Hartmann D., Thaddeus P., 2001, ApJ, 547, 792

Doane N. E., Sanders W. T., Wilcots E. M., Juda M., 2004, AJ, 128, 2712

Dottori H., Díaz R. J., Mast D., 2008, AJ, 136, 2468

Dottori H., Díaz R. J., Albacete-Colombo J. F., Mast D., 2010, ApJ, 717, L42 Dutta A., Sharma P., Nelson D., 2021, arXiv e-prints, p. arXiv:2107.02722

Farber R. J., Gronke M., 2021, arXiv e-prints, p. arXiv:2107.07991

Fielding D., Quataert E., Martizzi D., 2018, MNRAS, 481, 3325

Fielding D. B., Ostriker E. C., Bryan G. L., Jermyn A. S., 2020, ApJ, 894, L24

Foyle K., et al., 2012, MNRAS, 421, 2917

Frank Y., Mandelbaum P., Henis Z., 2014, High Energy Density Physics, 12, 27

Fujimoto Y., Bryan G. L., Tasker E. J., Habe A., Simpson C. M., 2016, MNRAS, 461, 1684

Gayley K. G., 2014, ApJ, 788, 90

Ge C., et al., 2015, ApJ, 812, 130
Giles P. A., et al., 2016, A\&A, 592, A3

Gissis I., Peretz U., Behar E., 2020, MNRAS, 492, 5634

Gronke M., Oh S. P., Ji S., Norman C., 2021, arXiv e-prints, p. arXiv:2107.13012

HI4PI Collaboration et al., 2016, A\&A, 594, A116

Hernandez S., et al., 2019, ApJ, 872, 116

Hobbs A., Feldmann R., 2020, MNRAS, 498, 1140

Hopkins P. F., Kereš D., Oñorbe J., Faucher-Giguère C.-A., Quataert E., Murray N., Bullock J. S., 2014, MNRAS, 445, 581

Hopkins P. F., Chan T. K., Squire J., Quataert E., Ji S., Kereš D., FaucherGiguère C.-A., 2021, MNRAS, 501, 3663

Jarrett T. H., et al., 2013, AJ, 145, 6

Jarrett T. H., Cluver M. E., Brown M. J. I., Dale D. A., Tsai C. W., Masci F., 2019, ApJS, 245, 25

Jeffreson S. M. R., Krumholz M. R., Fujimoto Y., Armillotta L., Keller B. W., Chevance M., Kruijssen J. M. D., 2021, arXiv e-prints, p. arXiv:2105.11457

Jiang X., Li J., Fang T., Wang Q. D., 2019, ApJ, 885, 38

Kannan R., Vogelsberger M., Marinacci F., Sales L. V., Torrey P., Hernquist L., 2021, MNRAS, 503, 336

Kelly A. J., Jenkins A., Frenk C. S., 2020, arXiv e-prints, p. arXiv:2005.12926

Kim C.-G., et al., 2020, ApJ, 900, 61

Koda J., et al., 2020, ApJ, 890, L10

Kuno N., et al., 2007, PASJ, 59, 117

Kuntz K. D., Snowden S. L., 2010, ApJS, 188, 46

Lada C. J., Lada E. A., 2003, ARA\&A, 41, 57

Lancaster L., Ostriker E. C., Kim J.-G., Kim C.-G., 2021, ApJ, 914, 89

Lehmer B. D., et al., 2017, ApJ, 851, 11

Leitherer C., et al., 1999, ApJS, 123, 3

Leroy A. K., Walter F., Brinks E., Bigiel F., de Blok W. J. G., Madore B., Thornley M. D., 2008, AJ, 136, 2782

Leroy A. K., et al., 2021, arXiv e-prints, p. arXiv:2104.07739

Li J.-T., Wang Q. D., 2013, MNRAS, 428, 2085

Li H., Vogelsberger M., Marinacci F., Sales L. V., Torrey P., 2020, MNRAS, 499,5862

Lingenfelter R. E., 2019, ApJS, 245, 30

Liu G., et al., 2013, ApJ, 778, L41

Long K. S., Kuntz K. D., Blair W. P., Godfrey L., Plucinsky P. P., Soria R., Stockdale C., Winkler P. F., 2014, ApJS, 212, 21

Lundgren A. A., Olofsson H., Wiklind T., Rydbeck G., 2004, A\&A, 422, 865

Mac Low M.-M., McCray R., 1988, ApJ, 324, 776

Maddox L. A., Cowan J. J., Kilgard R. E., Lacey C. K., Prestwich A. H., Stockdale C. J., Wolfing E., 2006, AJ, 132, 310

Marasco A., Fraternali F., Binney J. J., 2012, MNRAS, 419, 1107

McCray R., Kafatos M., 1987, ApJ, 317, 190

Mineo S., Gilfanov M., Sunyaev R., 2012, MNRAS, 426, 1870

Mosenkov A. V., et al., 2018, A\&A, 616, A120

Nakashima S., Inoue Y., Yamasaki N., Sofue Y., Kataoka J., Sakai K., 2018, ApJ, 862, 34

Owen R. A., Warwick R. S., 2009, MNRAS, 394, 1741

Peek J. E. G., Heiles C., Putman M. E., Douglas K., 2009, ApJ, 692, 827

Poetrodjojo H., D’Agostino J. J., Groves B., Kewley L., Ho I. T., Rich J., Madore B. F., Seibert M., 2019, MNRAS, 487, 79

Portegies Zwart S. F., McMillan S. L. W., Gieles M., 2010, ARA\&A, 48, 431

Quataert E., Thompson T. A., Jiang Y.-F., 2021, arXiv e-prints, p. arXiv:2102.05696

Röhser T., Kerp J., Lenz D., Winkel B., 2016, A\&A, 596, A94

Shabani F., et al., 2018, MNRAS, 478, 3590

Shetty R., Ostriker E. C., 2006, ApJ, 647, 997

Smith R. K., Brickhouse N. S., Liedahl D. A., Raymond J. C., 2001, ApJ, 556, L91

Somerville R. S., Davé R., 2015, ARA\&A, 53, 51

Soria R., Wu K., 2003, A\&A, 410, 53

Tang S., Wang Q. D., 2005, ApJ, 628, 205

Tang S., Wang Q. D., Lu Y., Mo H. J., 2009, MNRAS, 392, 77

Thim F., Tammann G. A., Saha A., Dolphin A., Sandage A., Tolstoy E., Labhardt L., 2003, ApJ, 590, 256 
Tyler K., Quillen A. C., LaPage A., Rieke G. H., 2004, ApJ, 610, 213 Vargas C. J., et al., 2018, ApJ, 853, 128

Vijayan A., Li M., 2021, arXiv e-prints, p. arXiv:2102.11510

Vijayan A., Kim C.-G., Armillotta L., Ostriker E. C., Li M., 2020, ApJ, 894, 12

Wall W. F., et al., 2016, MNRAS, 459, 1440

Wang Q. D., 2004, ApJ, 612, 159

Wang Q. D., 2010, Proceedings of the National Academy of Science, 107, 7168

Wang Q. D., Immler S., Walterbos R., Lauroesch J. T., Breitschwerdt D., 2001, ApJ, 555, L99

Wang Q. D., Li J., Jiang X., Fang T., 2016, MNRAS, 457, 1385

Weżgowiec M., Ehle M., Soida M., Dettmar R. J., Beck R., Urbanik M., 2020, A\&A, 640, A109

Wu R., et al., 2015, A\&A, 575, A88

van de Voort F., Quataert E., Hopkins P. F., Faucher-Giguère C.-A., Feldmann R., Kereš D., Chan T. K., Hafen Z., 2016, MNRAS, 463, 4533

van de Voort F., Bieri R., Pakmor R., Gómez F. A., Grand R. J. J., Marinacci F., 2021, MNRAS, 501, 4888

\section{APPENDIX A: DISTINCT EXTENDED BACKGROUND X-RAY SOURCES}

Although such background sources may be interesting in their own rights, we here probe their properties mainly to minimize their confusion with the diffuse X-ray emission intrinsic to M83 and to explore their potential utility in the study of the galaxy.

The background cluster of galaxies mentioned in the main text is apparent at R.A. $=13: 37: 08$, Dec. $=-29: 53: 40$ (Fig. 3A), although no identification in other wavelength bands is yet known (Long et al. 2014). The spectrum extracted from a radius of 25!'7 around the position can be well fitted with an APEC plasma $\left(\chi^{2} /\right.$ dof $\left.=562.85 / 515\right)$. This fit gives a characteristic temperature of $2.7_{-0.3}^{+0.5} \mathrm{keV}$ and a redshift of $0.56_{-0.01}^{+0.01}$, as well as a foreground absorption of a column density $N_{H}=2.7_{-0.7}^{+0.7} \times 10^{21} \mathrm{~cm}^{-2}$. This column density is consistent with the $\mathrm{N}_{\mathrm{HI}}+2 \mathrm{~N}_{\mathrm{H}_{2}}$ value of $\mathrm{N}_{H I} \approx 8 \times 10^{20} \mathrm{~cm}^{-2}$ obtained from the $21 \mathrm{~cm}$ line observation (Wall et al. 2016) and $\mathrm{N}_{H_{2}} \approx 2 \times 10^{21} \mathrm{~cm}^{-2}$ from $\mathrm{CO}(1-0)$ emission (Kuno et al. 2007), using the Galactic conversion factor $X_{\mathrm{CO}}=N_{\mathrm{H}_{2}} / I_{\mathrm{CO}}=$ $2 \times 10^{20} \mathrm{~cm}^{-2}\left[\mathrm{~K} \mathrm{~km} \mathrm{~s}^{-1}\right]^{-1}$ (Dame et al. 2001). This consistency indicates no significant $\mathrm{H}_{2}$ without $\mathrm{CO}$ emission along the line of sight. Adopting the best-fit redshift, we infer a $0.5-2.0 \mathrm{keV}$ luminosity of $3.1 \times 10^{43} \mathrm{erg} \mathrm{s}^{-1}$, consistent with the X-ray luminositytemperature scaling relation of galaxy clusters (e.g., Giles et al. 2016).

On the opposite side of the background cluster relative to the nucleus of M83 is a background FR II radio galaxy, which consists of a radio core R-28 and its radio lobes on both sides (e.g., Fig. A1; Cowan et al. 1994; Maddox et al. 2006). This radio core spatially coincides with a point-like X-ray source (XS-39 in Soria \& Wu 2003). Marginally detected at the position of this source is $\mathrm{H} \alpha$ line emission with a receding velocity of $\sim 130 \mathrm{~km} \mathrm{~s}^{-1}$, as was reported by Dottori et al. (2008). They further claimed the detection of an $\mathrm{Fe}$ emission line in the Chandra spectrum of the source with a small velocity shift corresponding to $z=0.018$. It was then proposed that the source might be associated with M83 (Dottori et al. 2010). In this scenario, R-28/XS-39 would represent a black hole kicked-off from the nucleus of M83 and could have observational effect on the ISM in the galaxy. Indeed, the scenario has been invoked in the explanation of the pressure gradient observed in molecular gas observed in the region of M83 (Wu et al. 2015).

We here critically examine the scenario with the deep Chandra

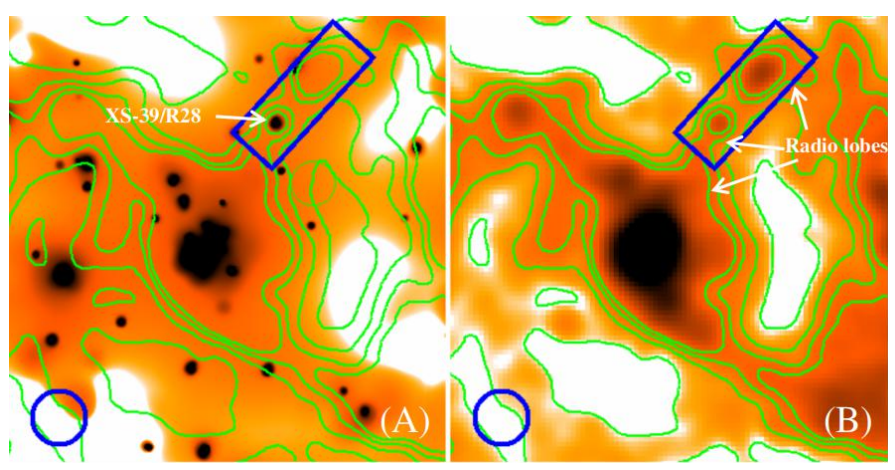

Figure A1. Close-ups of the M83 central field, including the background FR II radio galaxy (enclosed within the blue box): (A) a Gaussian smoothed Chandra intensity image in the $0.45-1 \mathrm{keV}$ band; (B) 6-cm continuum intensity image (Cowan et al. 1994). Overlaid in both panels are the radio intensity contours at 3,5,7, and $10 \sigma$ above the background. The lower left blue circle marks a half kpc diameter scale.

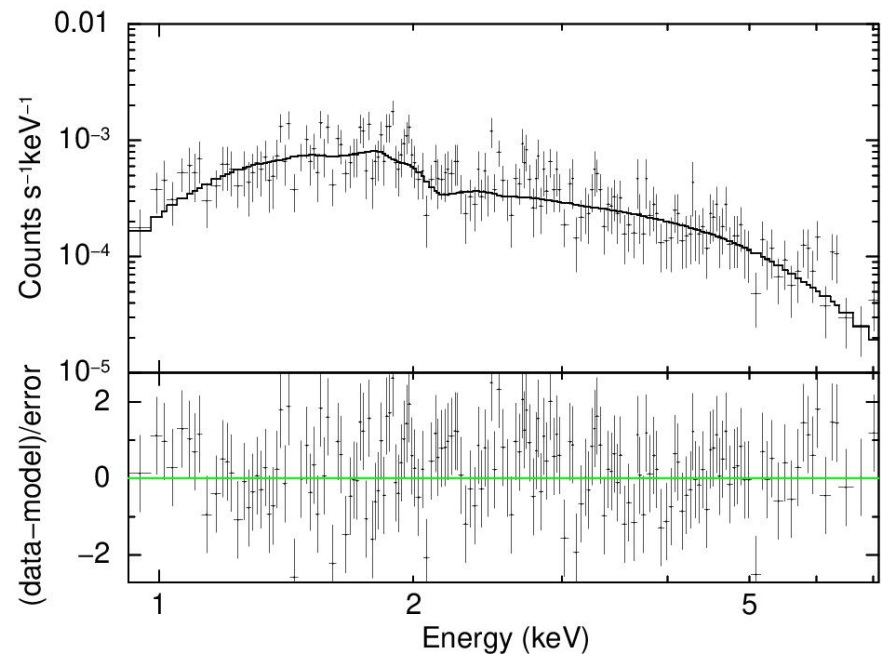

Figure A2. ACIS-S3 spectrum of the background FR II radio galaxy, together with the best-fit power law spectrum.

observations presented here. The Chandra spectrum used by Dottori et al. (2010) is from one observation of exposure 51 ks taken in 2000. With a total of only 135 net detected counts, their claimed presence of the Fe line is assumed to be intrinsically at $6.7 \mathrm{keV}$. Our analysis does not confirm the presence of the line, both statistically and visually (especially on the linear flux density scale, instead of the logarithmic one used in Dottori et al. (2010)). The lack of a significant $\mathrm{Fe} \mathrm{K} \alpha$ line in the spectrum of CS-39 is confirmed by our spectral analysis based on the accumulated data from the deep Chandra observations (e.g., Fig. A2). We confirm the position coincidence of XS-39 with R-28. The X-ray association with the radio galaxy may even be more extended (e.g., toward to the SE; Fig. C1). We speculate that the inverse Compton scattering of radio lobes may be responsible for both the linear morphology and the enhanced diffuse and relatively hard X-ray emission (see Fig. A1).

One may expect a large Faraday rotation of the radio lobes produced by line-of-sight magnetized ionized gas associated with M83. In principle, by mapping the Faraday rotation across the radio lobes, one could infer the spatial variation of the line-of-sight magnetic field in the galaxy. 


\section{APPENDIX B: HARDNESS RATIO CALCULATION}

This calculation of the hardness ratio (HR) is based on the X-ray intensities in the two bands (e.g., $S_{0.45-0.7}$ and $S_{0.7-1.0}$. The fluxes are both background-subtracted and exposure-corrected. HR and its error are then calculated as follows:

$$
\begin{aligned}
S & =\frac{C-B}{E} \\
\delta S & =\frac{\sqrt{C}}{E} \\
H R & =\frac{S_{0.7-1.0}}{S_{0.45-0.7}} \\
\delta H R & =\sqrt{\left(\frac{\delta S_{0.7-1.0}}{S_{0.45-0.7}}\right)^{2}+\left(H R \times \frac{\delta S_{0.45-0.7}}{S_{0.45-0.7}}\right)^{2}}
\end{aligned}
$$

where $C, B$ and $E$ are the detected counts, estimated background (including both the non-X-ray (stowed) and local sky X-ray contributions) and exposure (including the energy-dependent effective area of the telescope plus instrument) in each band. The small uncertainty in $B$, which is globally estimated, is neglected in the error propagation.

\section{APPENDIX C: SPECTRAL PARAMETER MAPPING}

We here first describe the procedure to produce maps of spectral parameters. We adaptively determine an extraction circle centered on each pixel to make sure that its spectrum has a signal to noise ratio $S / N \gtrsim 10$. This is accomplished with the software ClusterPyXT, which uses the blank sky background to approximately account for the background contribution to the noise (Alden et al. 2019). Our spectral mapping is limited to those pixels with the extraction circle radius less than $50^{\prime \prime}$. Each extraction includes the calculation of the local effective area as a function of photon energy to produce the effective area file (arf), while a single spectral matrix file ( $r m f)$ is adopted, since it varies little across the field. The spectra are then fitted individually with an adopted on-source spectral model to produce the spectral parameter maps.

By adopting the lognormal plasma plus power law model, we map out its spectral parameters in Fig. C1. They include the lognormal temperature dispersion, the normalization of the plasma, the power law normalization, goodness-of-the-fit $\left(\chi^{2}\right.$-dof) $/ \sqrt{2 \text { dof }}$ (under the normal approximation of the $\chi^{2}$ probability distribution). As in the main text, we fix $\bar{T}$ and $\Gamma$. We further fix the foreground X-ray absorption with a single representative intrinsic absorption column density or its approximate mean value of $N_{H}=1 \times 10^{21} \mathrm{~cm}^{-2}$ (Table 1), plus the Galactic absorption, to minimize the degeneracy that a freely fitting $N_{H}$ would introduce, especially when the counting statistics of the individual spectra in the mapping is poor.

The interpretation of these maps is tricky because of the degeneracy among the model parameters. The plasma normalization, for example, is strongly anti-correlated with $\sigma_{x}$, which partly explains the relatively small normalization at the leading edge of the southeast spiral arm, where the X-ray intensity is high. Another potential cause for this apparent discrepancy is the assumed constant foreground absorption. The actual absorption may be significantly higher in the region southwest of the galactic center, as well as at the leading edges of the spiral arms. Adopting a more realistic absorption, the normalization would be higher. We decide not to increase the modeling complexity in the spectral parameter mapping. Fundamentally, better data are needed to improve the constraints on the spectral properties of the X-ray emission from M83. 

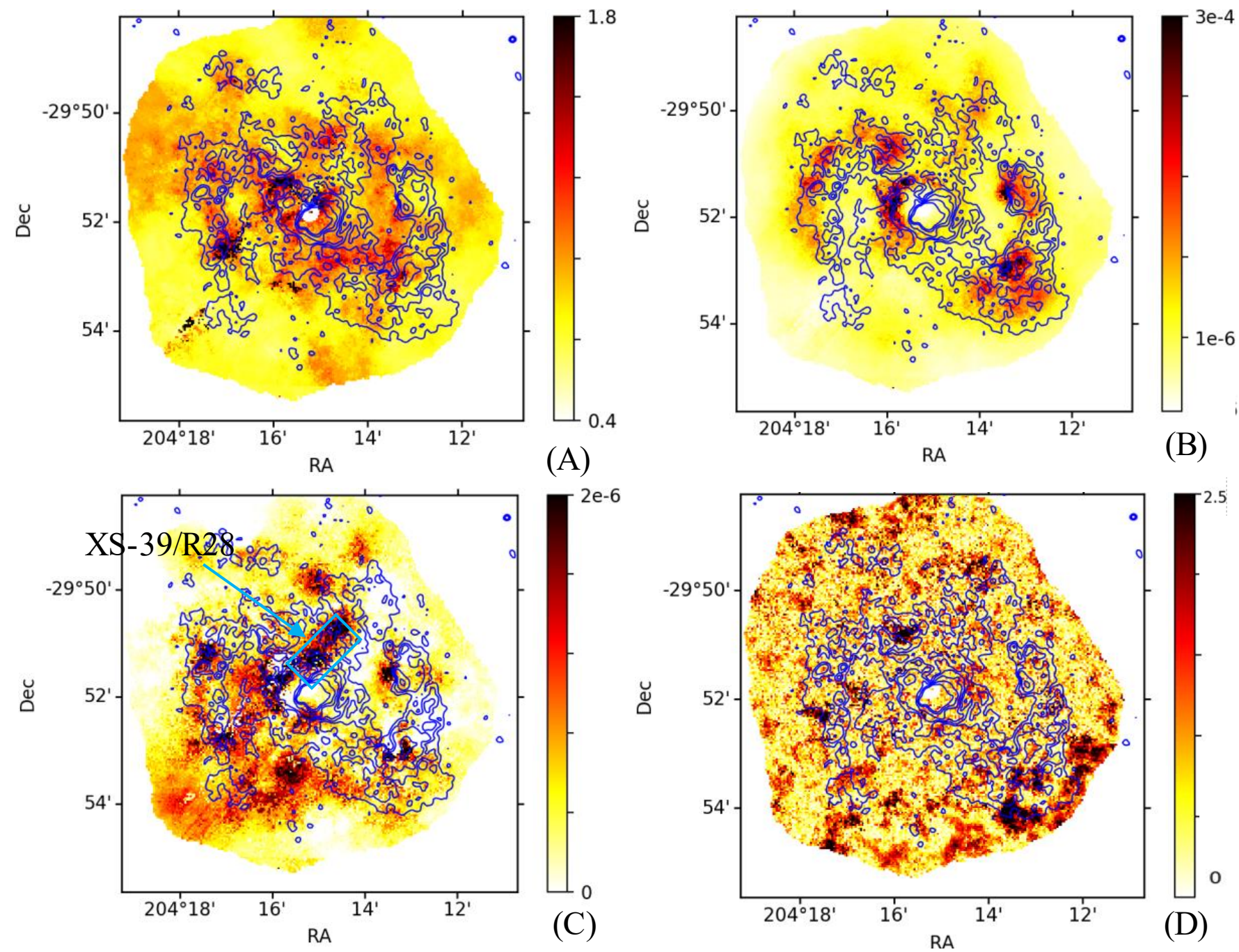

Figure C1. Maps of the spectral parameters: (A) the lognormal temperature dispersion $\sigma_{x}$; (B) the normalization of the plasma, scaled to the median spectral extraction area of $0.668 \operatorname{arcmin}^{2}$; (C) the power law normalization with the region of the background radio galaxy outlined (see also Fig. A1); and (D) the

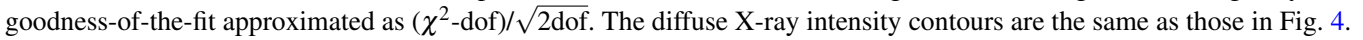

